\title{
REFINEMENT PROPERTIES AND EXTENSIONS OF FILTERS IN BOOLEAN ALGEBRAS
}

\author{
BY
}

BOHUSLAV BALCAR, PETTR SIMON AND PETER VOJTÁS

\begin{abstract}
We consider the question, under what conditions a given family $A$ in a Boolean algebra $B$ has a disjoint refinement. Of course, $A$ cannot have a disjoint refinement if $A$ is a dense subset of an atomless $\mathscr{B}$, or if $\mathscr{B}$ is complete and $A$ generates an ultrafilter on $\mathscr{B}$. We show in the first two sections that these two counterexamples can be the only possible ones. The third section is concerned with the question, how many sets must necessarily be added to a given filter in order to obtain an ultrafilter base.
\end{abstract}

0. Introduction. Let us recall the famous Disjoint Refinement Lemma due to Bernstein, Kuratowski, Sierpiński and others: "Assume $\kappa$ to be an infinite cardinal and let $A=\left\{a_{\alpha}: \alpha<\kappa\right\}$ be a family of sets, each of power $\kappa$. Then there is a family $D=\left\{d_{\alpha}: \alpha<\kappa\right\}$ such that for every $\alpha<\beta<\kappa$ we have $\left|d_{\alpha}\right|=\kappa, d_{\alpha} \subseteq a_{\alpha}, d_{\alpha} \cap d_{\beta}$ $=\varnothing$. The family $D$ is called a disjoint refinement of the family $A$.

This lemma, first conceived as a mere technical tool, has turned out to be the birth cry of the following general disjoint refinement problem: "What are the conditions under which a given family has a disjoint refinement?" The power set of a given set, a factor algebra of a set modulo some ideal, a partially ordered set and most generally, the Boolean algebra, for all these structures the question is meaningful. The problem has an extensive literature (e.g. [B], [BF], [BHM], [BV], $\left.[\mathbf{C}],[\mathbf{C H}],\left[\mathbf{v D}_{\mathbf{2}}\right],[\mathbf{H}],[\mathbf{H i}],[\mathbf{K}],\left[\mathbf{K u}_{\mathbf{2}}\right],[\mathbf{K r}],[\mathbf{P}],[\mathbf{S i}],[\mathbf{T}]\right) ;$ the results concerning the problem have plenty of applications-in Boolean algebras, in the theory of filters and ultrafilters, in the theory of ultrapowers, in the descriptive set-theory and topology.

The aim of the present paper is to study the refinement problem in Boolean algebras. The notion of the disjoint refinement is the natural one.

0.1. Definition. Let $\mathscr{B}$ be a Boolean algebra, $\kappa$ an infinite cardinal and $A=\left\{a_{\alpha}: \alpha<\kappa\right\}$ a family of nonzero elements of $\mathscr{B}$. The family $A$ has a disjoint refinement in $\mathscr{B}$ if there is a family $D=\left\{d_{\alpha}: \alpha<\kappa\right\}$ such that for each $\alpha<\beta<\kappa$ we have $\mathbf{0} \neq d_{\alpha} \leqslant a_{\alpha}$ and $d_{\alpha} \wedge d_{\beta}=\mathbf{0}$.

The paper is organized as follows: $\S 1$ is devoted to the refinement property for an arbitrary family $\boldsymbol{A}$. To make the paper self-contained, three facts from $\left[\mathbf{B V}_{1}\right]$ are quoted with proofs here. We shall state in terms of cardinal characteristics for

Received by the editors March 12, 1980.

1980 Mathematics Subject Classification. Primary 04A20, 06E05; Secondary 06E10, 54A25, 54G05.

Key words and phrases. Boolean algebra, disjoint refinement, ultrafilter, distributivity, Stone space, Gleason space.

(C) 1981 American Mathematical Society 0002-9947/81/0000-0418/\$05.75 
Boolean algebras which properties of $\mathscr{B}$ imply that $A$ has a disjoint refinement. The main results of this section are stated in Theorems 1.5 and 1.12. Besides, some technical tools are built up here.

The disjoint refinement property for centered families is studied in \$2. Perhaps the typical special cases will best illustrate the spirit of this section: Let $\mathscr{B}=$ $\mathscr{P}(\omega)$ /fin, $\tilde{\mathscr{B}}$ its completion. In other words, $\mathscr{B}$ is the algebra of all clopen subsets of $\beta N-N, \tilde{\mathscr{B}}$ is the algebra of all regular open subsets of $\beta N-N$. It is well known that $|\mathscr{B}|=2^{\omega},|\tilde{B}|=2^{2^{\omega}}$, and every maximal disjoint set in $\mathscr{B}$ as well as in $\widetilde{\mathscr{B}}$ is of cardinality $\leqslant 2^{\omega}$. Thus no family of cardinality $>2^{\omega}$ in $\tilde{\mathscr{B}}$ can have a disjoint refinement; on the other hand, each family of cardinality $<2^{\omega}$ in $\mathscr{B}$ or in $\tilde{\mathscr{B}}$ has one. Trivially, $\mathscr{B}-\{\boldsymbol{0}\}$ is a family of cardinality $2^{\omega}$ having no disjoint refinement both in $\mathscr{B}$ and in $\mathscr{B}$. According to $\left[\mathbf{B V}_{\mathbf{2}}\right]$, each ultrafilter in $\mathscr{B}$ has a disjoint refinement, consequently each centered family in $\mathscr{B}$ has a disjoint refinement. It turns out that things are different for $\tilde{\mathscr{B}}$. Obviously no ultrafilter base in $\tilde{\mathscr{B}}$ has a disjoint refinement, because $\tilde{\mathscr{B}}$ is complete. Thus if we ask the question, whether each centered family of cardinality $2^{\omega}$ in $\tilde{B}$ has a disjoint refinement, we must know that such a family cannot generate an ultrafilter. It turns out that this necessary condition need not be satisfied, which happens, e.g., if $2^{\omega}=\omega_{1}$. We shall show that the statement "Each centered family in $\widetilde{\mathscr{B}}$ of cardinality $2^{\omega}$ has a disjoint refinement" is both consistent with and independent of the usual axioms of set theory.

Fodor's conjecture [BHM] states that every family consisting of $\omega_{1}$ stationary sets in $\omega_{1}$ has a disjoint refinement by stationary sets. We shall show the following: If there is no $\omega_{1}$-scale in ${ }^{\omega} \omega$, Fodor's conjecture is true for "centered collections". On the other hand, under $(\mathrm{CH})$, the negation of Fodor's conjecture holds if and only if there is a family $Y$ of $\omega_{1}$ sets such that all closed unbounded subsets of $\omega_{1}$ together with the family $Y$ generate an ultrafilter on $\omega_{1}$.

These results indicate that there is a close connection between the disjoint refinement property and extensions of filters. In $\$ 3$, we shall study the problem, how many sets must be added to a given filter in order to obtain an ultrafilter. The main result states that for each infinite $\kappa$ and for each regular $\lambda, \omega_{1} \leqslant \lambda \leqslant 2^{\kappa}$, there is a filter $F$ and a family $X=\left\{x_{\alpha}: \alpha \in \lambda\right\}$ such that $F \cup X$ generates a uniform ultrafilter $U$ on $\kappa$. Moreover, $\lambda$ is the least cardinality of a family $Y$ such that $F \cup Y$ generates $U$.

ACKNOWLEDGEMENT. We would like to thank Richard Laver for his valuable information on recent results and stimulating discussions.

0.2. Notation. We shall use the standard set-theoretical notation to be found, e.g., in $[\mathbf{J}]$ or $[\mathbf{C N}]$. Small Greek letters $\kappa, \lambda$ always denote cardinal numbers. $\mathscr{P}(\kappa)$ is the power set of $\kappa,[\kappa]^{\lambda}=\{M \in \mathscr{P}(\kappa):|M|=\lambda\}$, similarly, $[\kappa]^{<\lambda}=\{M \in \mathscr{P}(\kappa)$ : $|M|<\lambda\}$. The weak power of cardinals, $\kappa^{\lambda}$, as usual, equals $\Sigma\left\{\kappa^{\alpha}: \alpha\right.$ is a cardinal, $\alpha<\lambda\}$. The Fréchet ideal on $\kappa$, denoted by $i_{F}$, equals $[\kappa]^{<\kappa}$.

Let $\mathscr{B}$ be a Boolean algebra. Operations on $\mathscr{B}$ are denoted $\vee$ (join), $\wedge$ (meet), - (complement), $\mathbf{0}$ or $\mathbf{0}_{\mathscr{B}}$ is the zero element of $\mathscr{B} \cdot \mathscr{B}^{+}=\mathscr{B}-\{\boldsymbol{0}\}$ is the set of all nonzero elements of $\mathscr{B}$. For $u \in \mathscr{B}^{+}, \mathscr{B} \uparrow u$ is the Boolean algebra consisting of 
all $v \leqslant u$ with relativized operations. $\delta \mathrm{t}(\mathscr{B})$ denotes the Stone space of an algebra $\mathscr{B}$. If $X$ is a topological space, then $R O(X)$ denotes the (complete) Boolean algebra of all regular open subsets of $X, G(X)=\delta \mathrm{t}(R O(X))$ is the absolute of $X$ (i.e. the Gleason space of $X=$ the projective resolution of $X$ ). If $\mathscr{B}$ is a Boolean algebra, $\operatorname{Comp}(\mathscr{B})$ is its completion. A subset $C \subseteq \mathscr{B}^{+}$is said to be dense in $\mathscr{B}$, or to be a base of $\mathscr{B}$, if for each $u \in \mathscr{B}^{+}$there is some $x \in C$ with $x \leqslant u$. A family (set, collection) $D \subseteq \mathscr{B}^{+}$is said to be disjoint if $d \wedge d^{\prime}=\mathbf{0}$ for any two distinct members $d, d^{\prime}$ from $D$. The density of $\mathscr{B}$ is defined by $d(\mathscr{B})=\min \{|C|: C$ is a dense subset of $\mathscr{B}\}$, the saturatedness of $\mathscr{B}$, $\operatorname{sat}(\mathscr{B})=\min \{\kappa$ : for each disjoint $\left.P \subseteq \mathscr{B}^{+},|P|<\kappa\right\}$. Hereditary analogues of these cardinal invariants are defined by hd( $(\mathscr{B})=\min \{d(\mathscr{B} \uparrow u): u \neq 0\}$ and hsat( $(\mathscr{B})=\min \{\operatorname{sat}(\mathscr{B} \uparrow u): u \neq 0\}$.

Let $\lambda \geqslant \omega, \kappa \geqslant 2$ be cardinals. Consider the family $C(\lambda, \kappa)=\{f: f$ is a mapping, $\left.\operatorname{dom}(f) \in[\lambda]^{<\lambda}, \operatorname{rng}(f) \subseteq \kappa\right\}$ with the order $f \geqslant g$ iff $f \subseteq g$. The (unique) complete Boolean algebra containing $C(\lambda, \kappa)$ as a dense subset is denoted by $\operatorname{Col}(\lambda, \kappa)$. If $u \in \mathscr{B}, P, Q \subseteq \mathscr{B}$, we shall denote $u \wedge \wedge P=\{u \wedge v: v \in P \& u \wedge v \neq 0\}$; similarly,

$$
P \wedge \wedge Q=\{u \wedge v: u \in P, v \in Q \& u \wedge v \neq 0\} .
$$

1. Refinement properties. Here we state the basic theorems on refinement properties of Boolean algebras.

1.1. Definition. Let $\mathscr{B}$ be a Boolean algebra, let $A \subseteq \mathscr{B}^{+}$. The set $A$ is called $\kappa$-decomposable if there exists a disjoint collection $P \subseteq \mathfrak{B}^{+}$such that $|P|=\mid a \wedge$ $\wedge P \mid=\kappa$ for each $a \in A$.

The following proposition is folklore:

1.2. Proposition. Let $\mathscr{B}$ be a Boolean algebra, $A=\left\{a_{\alpha}: \alpha \in \kappa\right\} \subseteq \mathscr{B}^{+}$. If $A$ is $\kappa$-decomposable, then $A$ has a disjoint refinement.

Proof. Let $P$ be some disjoint collection with $\left|a_{\alpha} \wedge \wedge P\right|=\kappa$ for each $\alpha \in \kappa$. By a straightforward recursion argument one can assign to each $\xi \in \kappa$ a member $x_{\xi} \in P$ such that $x_{\xi} \wedge x_{\eta}=\mathbf{0}$ and $a_{\xi} \wedge x_{\xi} \neq \mathbf{0}$ whenever $\xi<\eta<\kappa$. The family $D=\left\{a_{\xi} \wedge x_{\xi}: \xi \in \kappa\right\}$ is the desired disjoint refinement of $A$.

1.3. Definition. Let $\mathscr{B}$ be a Boolean algebra, $\kappa$ a cardinal. The algebra $\mathscr{B}$ has a disjoint refinement property for systems of cardinality at most $\kappa(\mathscr{B}$ has $\operatorname{Rp}(\kappa))$ if each $A=\left\{a_{\xi}: \xi \in \kappa\right\} \subseteq \mathscr{B}^{+}$has a disjoint refinement.

1.4. Remarks. (a) Clearly an algebra $\mathscr{B}$ has $\operatorname{Rp}(\kappa)$ iff $\operatorname{Comp}(\mathscr{B})$ has.

(b) If $\mathscr{B}$ has $\operatorname{Rp}(\kappa)$, then hsat $(\mathscr{B}) \geqslant \kappa^{+}$.

(c) If hsat $(B)>\kappa$, then there is no essential difference between the indexed families of cardinality $\kappa$ in $\mathscr{B}$ and sets of cardinality $\kappa$ in $\mathscr{B}$ from the refinement property point of view.

(d) If $\mathscr{B}$ has $\operatorname{Rp}(2)$, then $\mathscr{B}$ is atomless.

The following theorem has appeared in $\left[\mathbf{B V}_{1}\right]$ and generalizes a results of $[\mathbf{B H M}]$.

1.5. THEOREM. Let $\mathscr{B}$ be a Boolean algebra, $\kappa$ an infinite cardinal and let $\operatorname{hsat}(\mathscr{B})>\kappa^{+}$. Then $\mathscr{B}$ has a disjoint refinement property for systems of cardinality gt most $\kappa$. 
Proof. Assume given $A=\left\{a_{\xi}: \xi \in \kappa\right\} \subseteq \mathscr{B}^{+}$. Let $\Omega$ be the set of all disjoint sets $P$ in $\mathscr{B}^{+}$satisfying $|P| \geqslant \kappa^{+}$and either $\left|a_{\xi} \wedge \wedge P\right| \geqslant \kappa$ or $a_{\xi} \wedge \wedge P=\varnothing$ for each $\xi<\kappa$. Ordered by inclusion, $\Omega$ clearly satisfies the assumptions of the maximality principle. Let $R$ be a maximal element in $\Omega$.

Claim. For each $\xi<\kappa,\left|a_{\xi} \wedge \wedge R\right| \geqslant \kappa$. Suppose the contrary. Then $a_{\xi} \wedge \wedge R$ $=\varnothing$ for some $\xi<\kappa$. Choose a disjoint family $Q$ in $\mathscr{B}\left|a_{\xi},\right| Q \mid=\kappa^{+}$. Let $Z=\left\{\eta \in \kappa:\left|a_{\eta} \wedge \wedge Q\right|<\kappa\right\}$, let $Q^{\prime}=Q-\left\{u \in Q: a_{\eta} \wedge u \neq 0\right.$ for some $\eta \in$ $Z$ \}. Then $\left|Q^{\prime}\right|=\kappa^{+}, R \cup Q^{\prime} \in \Omega, R \cup Q^{\prime} \supsetneqq R$, a contradiction.

Having proved the claim, notice that $R$ clearly contains a set which $\kappa$-decomposes $A$, hence 1.2 applies.

REMARKS. In fact, we have proved a bit more: If $A=\left\{a_{\alpha}: \alpha \in \kappa\right\} \subseteq \mathscr{B}^{+}$is such that $\operatorname{sat}\left(\mathscr{B} \uparrow a_{\alpha}\right)>\kappa^{+}$for each $\alpha \in \kappa$, then $A$ has a disjoint refinement.

The Disjoint Refinement Lemma, quoted in the introduction, can be quickly deduced from the previous theorem: If $\kappa$ is infinite and regular, then $\operatorname{hsat}\left(\mathscr{P}(\kappa) / i_{F}\right)$ $>\kappa^{+}$, thus 1.5 may be applied. If $\kappa$ is singular, then the Disjoint Refinement Lemma is easy to prove by making use of its validity for all regular cardinals smaller than $\kappa$.

Using 1.5, one may obtain the following strengthening of 1.2.

1.6. Lemma. Let $\mathscr{B}$ be a complete Boolean algebra, hsat $(\Re) \geqslant \kappa^{+}, A=\left\{a_{\alpha}\right.$ : $\alpha \in \kappa\} \subseteq \mathscr{B}^{+}$. Then $A$ has a disjoint refinement if and only if $A$ is $\operatorname{cf}(\kappa)$-decomposable.

Proof. Denote $\tau=\operatorname{cf}(\kappa)$. If $\kappa>\tau$, choose an arbitrary increasing sequence $\left\{\kappa_{\xi}\right.$ : $\xi \in \tau\}$ of cardinals converging to $\kappa$.

Suppose $A$ to be $\tau$-decomposable. By 1.2, only the case $\tau<\kappa$ has to be considered. Let $P=\left\{p_{\xi}: \xi \in \tau\right\}$ be a disjoint set satisfying $\left|a_{\alpha} \wedge \wedge P\right|=\tau$ for each $\alpha \in \kappa$. Then each family $C_{\xi}=\left\{a_{\alpha} \wedge p_{\xi}: \alpha<\kappa_{\xi} \& a_{\alpha} \wedge p_{\xi} \neq 0\right\}$ has a disjoint refinement $D_{\xi}$ by 1.5. Clearly $\cup\left\{D_{\xi}: \xi \in \tau\right\}$ contains a disjoint refinement of $A$. The completeness of $\mathscr{B}$ was not needed in this half of the proof.

Let $\left\{d_{\alpha}: \alpha \in \kappa\right\}$ be a disjoint refinement of $A$. Since hsat $(\mathscr{B}) \geqslant \kappa^{+}$, there is a pairwise disjoint set $\left\{d_{\alpha \xi}: \xi \in \tau\right\}$ in $\mathscr{B} \uparrow d_{\alpha}$ for each $\alpha \in \kappa$. The algebra $\mathscr{B}$ is complete, hence the set $P=\left\{\bigvee\left\{d_{\alpha \xi}: \alpha \in \kappa\right\}: \xi \in \tau\right\}$ is well defined and demonstrates that $A$ is $\tau$-decomposable.

1.7. LEMmA. Let $\mathscr{B}$ be a Boolean algebra, $\kappa$ infinite and $\mathrm{hd}(\mathscr{B}) \leqslant \kappa$. Then $\mathscr{B}$ has not $\operatorname{Rp}(\kappa)$.

Proof. Suppose the contrary. Let $\mathscr{B}$ have $\operatorname{Rp}(\kappa)$. Choose $x \in \mathscr{B}^{+}$such that $d(\mathscr{B} \uparrow x) \leqslant \kappa$, let $C \subseteq(\mathscr{B} \uparrow x)$ be dense in $\mathscr{B} \uparrow x$ and $|C| \leqslant \kappa$. Suppose $D$ is a disjoint refinement of $C$. Choose $d \in D$. Since $\mathscr{B}$ is atomless by $1.4(\mathrm{~d})$, and since $C$ is dense in $\mathscr{B} \uparrow x$ there is some $c \in C$ with $c \lessgtr d$. Clearly there is no $d^{\prime} \in D$ disjoint with $d$ and satisfying $d^{\prime} \leqslant c$.

Up to now we have dealt with an arbitrary Boolean algebra and we were interested in the problem whether an arbitrary $A=\left\{a_{\alpha}: \alpha \in \kappa\right\} \subseteq \mathscr{B}^{+}$has a disjoint refinement. Theorem 1.5 says that $\operatorname{hsat}(\mathscr{B})>\kappa^{+}$is a sufficient condition. 
Clearly hsat $(\mathscr{B})>\kappa$ is necessary (for otherwise there are not enough disjoint elements in $\mathscr{B}$ ). But Lemma 1.7 shows how to find an example that the last condition need not be sufficient. Thus, if we want to know something about the disjoint refinement property for systems $A=\left\{a_{\alpha}: \alpha \in \kappa\right\}$ which are as large as possible, i.e. $\kappa^{+}=\operatorname{hsat}(\mathscr{B})$, we have to add some restrictions either on $\mathscr{B}$ or on $A$. The first direction will be studied in the rest of this section and it will culminate in Theorem 1.12.

1.8. Definimion. Let $\mathscr{B}$ be a Boolean algebra, $C \subseteq \mathfrak{B}^{+}, \lambda$ an infinite cardinal. The set $C$ is $\lambda$-closed if for each $\gamma<\lambda$ and for each decreasing chain $c_{0}>c_{1}$ $\geqslant \cdots \geqslant c_{\alpha} \geqslant \cdots(\alpha<\gamma)$ of elements of $C$ there is some $b \in C$ with $b \leqslant c_{\alpha}$ for each $\alpha<\gamma$.

Remark. If $C \subseteq \mathscr{B}^{+}$is $\lambda$-closed for a singular cardinal $\lambda$, then $C$ is $\lambda^{+}$-closed.

1.9. Definition. Let $\mathscr{B}$ be a Boolean algebra, $\lambda, \kappa$ cardinals. A collection $\Theta \subseteq \mathscr{P}\left(\mathscr{B}^{+}\right)$is called a matrix on $\mathscr{B}$ if each member of $\Theta$ is a maximal disjoint subset of $\mathfrak{B}^{+}$.

The Boolean algebra $\mathscr{B}$ is called to be $(\lambda, \cdot, \kappa)$-distributive if for every matrix $\Theta=\left\{P_{\alpha}: \alpha \in \lambda\right\}$ there is some maximal disjoint system $Q \subseteq \mathscr{B}^{+}$such that for each $x \in Q$ and for each $\alpha \in \lambda,\left|x \wedge \wedge P_{\alpha}\right|<\kappa$. (The dot "." in $(\lambda, \cdot, \kappa)$ indicates we are not interested in the size of $P_{\alpha}$ 's. For the three-parameters distributivity see [VH].)

The Boolean algebra $\mathscr{B}$ is called $(\lambda, \cdot, \kappa)$-nowhere distributive if for every $x \in \mathscr{B}^{+}, \mathscr{B} \uparrow x$ is not $(\lambda, \cdot, \kappa)$-distributive.

A standard branching argument gives an immediate consequence of the definitions:

1.10. Lemma. If a Boolean algebra $\mathscr{B}$ has a $\lambda$-closed dense subset, then $\mathscr{B}$ is $(\tau, \cdot, 2)$-distributive for each $\tau<\lambda$.

1.11. Lemma. Let $\mathscr{B}$ be $a(\lambda, \cdot, \kappa)$-nowhere distributive Boolean algebra. Then there is a matrix $\Theta=\left\{P_{\alpha}: \alpha \in \lambda\right\}$ such that for each $x \in \mathscr{B}^{+}$there is some $\alpha \in \lambda$ with $\left|x \wedge \wedge P_{\alpha}\right| \geqslant \kappa$.

Any matrix with this property will be called a matrix witnessing to the $(\lambda, \cdot, \kappa)$ nowhere distributivity of $\mathscr{B}$.

Proof. Consider the set $C$ consisting of all $x \in \mathscr{B}^{+}$for which there is some matrix $\Theta(x)=\left\{P_{\alpha}(x): \alpha \in \lambda\right\}$ in $\mathscr{B} \mid x$ such that for each $y \leqslant x$ there is some $\alpha \in \lambda$ with $\left|y \wedge \wedge P_{\alpha}(x)\right| \geqslant \kappa$.

The set $C$ is dense in $\mathscr{B}$ : If not, assume $z \in \mathscr{B}^{+}$contains no member of $C$. Thus for each matrix $\left\{P_{\alpha}: \alpha \in \lambda\right\}$ in $\mathscr{B} \uparrow z$ the set $\left\{y \in \mathscr{B} \mid z:(\forall \alpha \in \lambda)\left(\left|y \wedge \wedge P_{\alpha}\right|\right.\right.$ $<\kappa)\}$ is dense in $\mathscr{B} \uparrow z$, hence there is a maximal disjoint set $Q$ in $\mathscr{B} \uparrow z$ with the property $\left(y \in Q, \quad \alpha \in \lambda \rightarrow\left|y \wedge \wedge P_{\alpha}\right|<\kappa\right)$, which contradicts the $(\lambda, \cdot, \kappa)$ nowhere distributivity of $\mathscr{B}$.

Let $S$ be a maximal disjoint subset of $C$. Since $C$ is dense in $\mathscr{B}, S$ is maximal disjoint in $\mathscr{B}$, too. For $x \in S$ let $\Theta(x)$ be as indicated above, and define $P_{\alpha}=\{u$ $\in \mathscr{B}: u \in P_{\alpha}(x)$ for some $\left.x \in S\right\}$. The matrix $\Theta=\left\{P_{\alpha}: \alpha \in \lambda\right\}$ has the required properties. 
The forthcoming Theorem 1.12 is the main theorem of this section. It shows that the presence of a $\lambda$-closed dense set and $(\lambda, \cdot, \kappa)$-nowhere distributivity of $\mathscr{B}$ are the possible restrictive conditions on $\mathscr{B}$ which guarantee the existence of the disjoint refinement in all cases, for which the only obvious obstruction, i.e. a too small base, does not take place. At the same time we shall demonstrate for $\lambda$ regular that the well-known example of a $(\lambda, \cdot, \kappa)$-nowhere distributive algebra with a $\lambda$-closed base, namely the algebra $\operatorname{Col}(\lambda, \kappa)$, is typical and plays the key role in the problem of refinement. The theorem generalizes a result from $\left[\mathbf{B V}_{\mathbf{i}}\right]$, which is mentioned here as a corollary.

In the sequel we assume $\lambda \geqslant \omega, \kappa \geqslant 2$.

1.12. TheOREM. Let $\mathscr{B}$ be $a(\lambda, \cdot, \kappa)$-nowhere distributive Boolean algebra having $a$ $\lambda$-closed dense subset. Then the following conditions are equivalent:

(a) $\mathscr{B}$ has $\operatorname{Rp}\left(\kappa^{\lambda}\right)$,

(b) $\mathrm{hd}(\mathscr{B})>\kappa^{\lambda}$.

Moreover, if $\mathscr{B}$ is complete, then both (a) and (b) are equivalent to:

(c) for each $x \in \mathscr{B}^{+}, \mathscr{B} \uparrow x$ is not isomorphic to $\operatorname{Col}(\lambda, \kappa)$;

(d) for each $x \in \mathscr{B}^{+}, \mathscr{B} \uparrow x$ is not isomorphic to $\operatorname{Col}\left(\lambda, \kappa^{\lambda}\right)$.

1.13. Corollary $\left[\mathrm{BV}_{1}\right]$. Let $\mathscr{B}$ be an $(\omega, \cdot, \kappa)$-nowhere distributive Boolean algebra. Then $\mathscr{B}$ has $\mathrm{Rp}(\kappa)$ if and only if $\mathrm{hd}(\mathscr{B})>\kappa$.

Before giving a proof, we shall exhibit some propositions of technical nature leading to a characterization of algebras $\operatorname{Col}(\lambda, \kappa)$.

1.14. Lemma. Let $\mathscr{B}$ be $a(\lambda, \cdot, \kappa)$-nowhere distributive Boolean algebra containing $a \lambda$-closed dense subset $C$. Then:

(i) $\operatorname{hsat}(\mathscr{B})>\kappa^{\lambda}$;

(ii) $\lambda$ is a regular cardinal.

Let $\mathscr{P}=\left\{P_{\alpha}: \alpha \in \lambda\right\}$ be an arbitrary matrix on $\mathscr{B}$. Then there is a matrix $\mathcal{Q}=\left\{Q_{\alpha}: \alpha \in \lambda\right\}$ such that:

(iii) 2 witnesses to the $(\lambda, \cdot, \kappa)$-nowhere distributivity of $\mathscr{B}$;

(iv) $\cup \mathcal{Q} \subseteq C$;

(v) for each $\alpha<\beta<\lambda, Q_{\beta}$ refines $Q_{\alpha}$ and $Q_{\beta}$ refines $P_{\beta}$;

(vi) for each $\alpha \in \lambda$ and for each $y \in Q_{\alpha}$,

$$
\left|\left\{x \in Q_{\alpha+1}: x \leqslant y\right\}\right| \geqslant \kappa^{\lambda}
$$

(vii) for each $y \in \mathscr{B}^{+}$;

$$
|\{x \in \bigcup \mathscr{Q}: x \wedge y \neq \mathbf{0}\}| \geqslant \kappa^{\lambda}
$$

Proof. (i) follows from (vi) and from the fact that the lemma may be relativized to each $\mathscr{B} \uparrow x, x \in \mathscr{B}^{+}$.

The cardinal $\lambda$ is infinite, for $\mathscr{B}$ is $(\lambda, \cdot, \kappa)$-nowhere distributive. If it were singular, then $C$ would be $\lambda^{+}$-closed (see the Remark following 1.8), hence by 1.10 , $\mathscr{B}$ would be $(\lambda, \cdot, 2)$-distributive-a contradiction.

The matrix 2 will be constructed by transfinite induction. 
Let $\mathcal{S}=\left\{S_{\alpha}: \alpha \in \lambda\right\}$ be a matrix witnessing to $(\lambda, \cdot, \kappa)$-nowhere distributivity of $\mathscr{B}$. Let $Q_{0} \subseteq C$ be a maximal disjoint family refining $P_{0} \wedge \wedge S_{0}$.

Let $\alpha<\lambda$ and suppose $Q_{\beta}(\beta<\alpha)$ have been defined. By $1.10, \mathscr{B}$ is $(\alpha, \cdot, 2)-$ distributive, hence there is a maximal disjoint collection $U_{\alpha}$ such that $U_{\alpha}$ refines $P_{\alpha}$ and $Q_{\beta}$ for all $\beta<\alpha$. Let $V_{\alpha}=U_{\alpha} \wedge \wedge S_{\alpha}$. For $x \in V_{\alpha}$ take a maximal family $R_{x} \subseteq C$ such that $R_{x}$ is disjoint and its cardinality is at least $\mu$, where $\mu$ is some suitable cardinal.

As yet we know that $\mu \geqslant \kappa$ (hsat $(\mathscr{B})>\kappa$ by 1.11); we shall show later that the choice $\mu \geqslant \kappa^{\lambda}$ is possible. Let $Q_{\alpha}=\cup\left\{R_{x}: x \in V_{\alpha}\right\}$.

Having constructed the matrix $\mathscr{2}=\left\{Q_{\alpha}: \alpha \in \lambda\right\}$, it is easy to verify that (iii), (iv) and (v) hold for 2 , moreover, for each $\alpha \in \lambda$ and for each $y \in Q_{\alpha}$,

$$
\left|\left\{x: x \in Q_{\alpha+1} \& x \leqslant y\right\}\right| \geqslant \mu \geqslant \kappa .
$$

Let $\delta<\lambda$ be a cardinal, let $y \in \cup 2$. Then $y \in Q_{\beta}$ for some $\beta<\lambda$. By the validity of (1) for each $\alpha<\lambda$, by (iv) and by the fact that $C$ is $\lambda$-closed, we obtain

$$
\left|\left\{x: x \in Q_{\beta+\delta} \& x \leqslant y\right\}\right| \geqslant \mu^{\delta} \geqslant \kappa^{\delta} .
$$

Thus $\operatorname{sat}(\mathscr{B} \mid y) \geqslant \kappa^{\lambda}$. We shall show that $\operatorname{sat}(\mathscr{B} \mid y) \geqslant\left(\kappa^{\lambda}\right)^{+}$.

If $\kappa^{\lambda}=\kappa^{\delta}$ for some $\delta<\lambda$, then $\operatorname{sat}\left(\mathscr{B}\lceil y) \geqslant\left(\kappa^{\delta}\right)^{+}\right.$by (2). If $\kappa^{\lambda}>\kappa^{\delta}$ for each $\delta<\lambda$, then $\kappa^{\lambda}$ is a limit cardinal and $\lambda=\operatorname{cf}\left(\kappa^{\lambda}\right) \leqslant \kappa^{\lambda}$. Two cases are possible: Either $\lambda<\kappa^{\lambda}$, but then $\operatorname{sat}\left(\mathscr{B}\lceil y) \geqslant\left(\kappa^{\lambda}\right)^{+}\right.$since the saturatedness cannot be a singular cardinal by a well-known theorem of Erdös and Tarski [ET], or $\lambda=\kappa^{\lambda}$. Let $y=c_{\beta}$, choose by induction a chain $c_{\beta} \geqslant c_{\beta+1} \geqslant \ldots \geqslant c_{\alpha} \geqslant \ldots$ for each $\alpha \in \lambda, \alpha \geqslant \beta$, such that $c_{\alpha} \in Q_{\alpha}$. By (1), the inequalities are sharp, hence $D=\left\{c_{\alpha}\right.$ $\left.-c_{\alpha+1}: \beta \leqslant \alpha<\lambda\right\} \subseteq \mathscr{B}^{+}$is a disjoint subset of $(\mathscr{B} \mid y)^{+}$, which implies that $\operatorname{sat}\left(\mathscr{B}\lceil y) \geqslant \lambda^{+}\right.$.

Thus $\mu \geqslant \kappa^{\lambda}$ could have been chosen in the construction of 2 , which proves (vi).

It remains to prove (vii). Let $y \in \mathscr{B}^{+}$. The desired family $\{x: x \in \cup \mathcal{2} \& x \wedge$ $y \neq 0\}$ can be found using a standard branching argument. For $\xi=0$, set $\alpha_{0}=0$, pick $x_{0} \in Q_{0}$ so that $x_{0} \wedge y \neq \mathbf{0}$, and pick $c_{0} \in C$ with $c_{0} \leqslant x_{0} \wedge y$. For $\xi<\lambda, \xi$ limit, assume that $f: \xi \rightarrow \kappa$. Since $C$ is $\lambda$-closed, there is some $c \in C$ such that $c \leqslant c_{f \nmid \eta}$ for all $\eta<\xi$. By (iii), there is some smallest $\alpha_{f}<\lambda$ such that there is a disjoint set $\left\{b_{\imath}: \iota<\kappa\right\} \subseteq Q_{\alpha_{y}}$ with $b_{\imath} \wedge c \neq 0$ for all $\iota<\kappa$. Clearly $\alpha_{f}>\alpha_{f\lceil\eta}$ for all $\eta<\xi$. Let $x_{f}=b_{0}$. Choose $c_{f} \in C$ with $c_{f} \leqslant c \wedge x_{f}$. Now suppose $\xi=\eta+1$ and $f$ : $\eta \rightarrow \kappa$. By (iii) there is a smallest $\alpha<\lambda$ such that there is a disjoint set $\left\{b_{\iota}: \iota<\kappa\right\}$ satisfying $b_{\imath} \wedge c_{f} \neq 0$ for all $\iota<\kappa,\left\{b_{\imath}: \iota<\kappa\right\} \subseteq Q_{\alpha}$. Clearly $\alpha>\alpha_{f}$. For each $\iota<\kappa$ let $\alpha_{f \cup\{(\eta, \imath)\}}=\alpha, x_{f \cup\{(\eta, \imath)\}}=b_{\imath}$, and choose $c_{f \cup\{(\eta, \imath)\}}<c_{f} \wedge b_{\imath}, c_{f \cup\{(\eta, l)\}} \in C$.

Hence for each $\xi<\lambda$ and for each $f: \xi \rightarrow \kappa$ we have found an element $x_{f} \in \cup \mathcal{Q}$ such that the following is true: $x_{f} \wedge y \neq 0, x_{f} \leqslant x_{f \mid \eta}$ for all $\eta<\xi$, and if $g: \xi \rightarrow \kappa$, $g \neq f$, then $x_{f} \wedge x_{g}=\mathbf{0}$.

Now (vii) easily follows and the proof is complete.

1.15. THEOREM. Let $\mathscr{B}$ be a complete $(\lambda, \cdot, \kappa)$-nowhere distributive Boolean algebra containing a $\lambda$-closed dense subset $C$. Let $d(\mathscr{B})=\kappa^{\lambda}$. Then $\mathscr{B}$ is isomorphic to $\operatorname{Col}\left(\lambda, \kappa_{-}^{\lambda}\right)$. 
Proof. Let $D$ be a dense subset of $\Re,|D|=\kappa^{\lambda}$. Let $\mathscr{R}=\left\{R_{\alpha}: \alpha \in \lambda\right\}$ be an arbitrary matrix satisfying 1.14(iii)-(vii). Using 1.14(vii), one can define a mapping $\varphi: D \rightarrow \cup \Re$ with $d \wedge \varphi(d) \neq \mathbf{0}$ for each $d \in D$ and $\varphi(d) \neq \varphi\left(d^{\prime}\right)$ whenever $d \neq d^{\prime}$. For $\alpha<\lambda$, let $P_{\alpha}$ be a maximal disjoint family containing all elements $\varphi(d) \wedge d, \varphi(d)-d$ for $\varphi(d) \in R_{\alpha}$, and all $x \in R_{\alpha}-\{\varphi(d): d \in D\}$. Apply 1.14 to the matrix $\mathscr{P}=\left\{P_{\alpha}: \alpha \in \lambda\right\}$, let $\mathcal{L}$ be the resulting matrix. Now $\cup \mathcal{Q}$ is a dense subset of $\mathscr{B}$. By 1.14(v), (vi), a verification of the existence of an isomorphism between $\cup \mathcal{Q}$ and the set $\left\{f \in C\left(\lambda, \kappa^{\lambda}\right): \operatorname{dom}(f) \neq \varnothing, \operatorname{dom}(f)\right.$ is a successor ordinal $\}$, which forms a dense subset of $\operatorname{Col}\left(\lambda, \kappa^{\lambda}\right)$, is routine.

1.16. Corollary. The algebras $\operatorname{Col}(\lambda, \kappa)$ and $\operatorname{Col}\left(\lambda, \kappa^{\lambda}\right)$ are isomorphic whenever $\lambda \geqslant \omega, \kappa \geqslant 2, \lambda$ is regular.

1.17. LeMmA. Let $\mathscr{B}$ be a $(\lambda, \cdot, \kappa)$-nowhere distributive Boolean algebra having $a$ $\lambda$-closed dense subset. If $\operatorname{hd}(\mathscr{B})>\kappa^{\lambda}$, then $\mathscr{B}$ has $\operatorname{Rp}\left(\kappa^{\lambda}\right)$.

Proof. Let $A \subset \mathscr{B}^{+},|A|=\kappa^{\lambda}$. Let $\mathscr{2}=\left\{Q_{\alpha}: \alpha \in \lambda\right\}$ be a matrix having properties 1.14(iii)-(vii). We may assume that $A \subset \cup \mathcal{2}$ (see the proof of 1.15).

Denote $P_{\alpha}=A \cap Q_{\alpha}$. If there is some $\tau<\lambda$ such that $P_{\alpha}=\varnothing$ for each $\alpha<\lambda$, $\alpha \geqslant \tau$, we are done $\left(P_{\tau+1}\right.$ can be used to show that $A$ is $\kappa^{\lambda}$-decomposable). Thus suppose $P_{\alpha} \neq \varnothing$ for each $\alpha<\lambda$. Let $C$ be a $\lambda$-closed dense subset of $\Re$.

We shall find a family of chains $\{c(x, \alpha): \alpha<\lambda\}, x \in A$, with the following properties:

(a) $c(x, 0) \leqslant x$;

(b) $c(x, \alpha) \in C$ for each $x \in A, \alpha \in \lambda$;

(c) if $x \in A, \alpha<\beta<\lambda$, then $c(x, \alpha) \geqslant c(x, \beta) \neq 0$;

(d) if $x \in P_{\alpha}, y \in A, y \neq x$, then $c(x, \alpha+1) \wedge c(y, \alpha+1)=0$.

Obviously the set $\left\{c(x, \alpha+1): x \in P_{\alpha}, \alpha \in \lambda\right\}$ will be the desired disjoint refinement of $\boldsymbol{A}$.

The construction of the chains goes by transfinite induction. Let $\beta<\lambda$ be an ordinal and suppose that $c(x, \alpha)$ have been defined for each $x \in A$ and each $\alpha<\beta$.

$\beta$ limit: Choose $c(x, \beta) \in C$ such that $\mathbf{0} \neq c(x, \beta) \leqslant c(x, \alpha)$ for each $\alpha<\beta$, $x \in A$. The $\lambda$-closedness of $C$ enables us to do this.

$\beta=\gamma+1$ : If $x \in P_{\alpha}, \alpha<\gamma$, let $c(x, \gamma+1)=c(x, \gamma)$. Let $x \in P_{\gamma}$. The family $\{c(y, \gamma): y \in A\}$ is too small for being dense in $\mathscr{B} \uparrow c(x, \gamma)$, thus there is some $c(x, \gamma+1) \in C$ with $\mathbf{0} \neq c(x, \gamma+1) \leqslant c(x, \gamma)$ and $c(y, \gamma)-c(x, \gamma+1) \neq 0$ for each $y \in A$. Let $x \in P_{\alpha}, \alpha>\gamma$. By 1.14(v) there is at most one $y(x) \in P_{\gamma}$ such that $x \leqslant y(x)$; let $c(x, \gamma+1)$ be an arbitrary element from $C$ satisfying $\mathbf{0} \neq c(x, \gamma+1)$ $\leqslant c(x, \gamma)-c(y(x), \gamma+1)\left(\right.$ or $\mathbf{0} \neq c(x, \gamma+1) \leqslant c(x, \gamma)$ if no such $y(x) \in P_{\gamma}$ exists).

1.18. Proof of 1.12. Clearly (c) and (d) are equivalent (see 1.16). (a) implies (b) by 1.7 and (b) implies (a) is precisely the statement of 1.17 .

Notice that $\operatorname{hd}\left(\operatorname{Col}\left(\lambda, \kappa^{\lambda}\right)\right)=\kappa^{\lambda}$. This fact and 1.7 show that (a) implies (d).

To prove (d) implies (b), notice that if $\operatorname{hd}(\mathscr{B})=\kappa^{\lambda}$, if $\mathscr{B}$ is $(\lambda, \cdot, \kappa)$-nowhere distributive having a $\lambda$-closed dense subset and if $\mathscr{B}$ is complete, then $\mathscr{B}$ is isomorphic to $\operatorname{Col}\left(\lambda, \kappa^{\lambda}\right)$ by 1.15 . 
1.19. EXAMPLE. The assumption that $\mathscr{B}$ has a $\lambda$-closed dense subset is essential in 1.12. To show this, let $\kappa$ be a cardinal satisfying $\kappa^{\omega}=\kappa$. Then there is an $\left(\omega_{1}, \cdot, \kappa\right)$-nowhere distributive Boolean algebra $\mathscr{B}$ with $\mathrm{hd}(\mathscr{B})>\kappa\left(=\kappa \omega_{1}\right)$, nevertheless $\operatorname{Rp}(\kappa)$ does not hold for $\mathscr{B}$.

Let $F=\left\{f \in{ }^{\alpha} \kappa: \alpha<\omega_{1}\right\} . F$ is a dense subset of $\operatorname{Col}\left(\omega_{1}, \kappa\right),|F|=\left(\kappa \times \omega_{1}\right)^{\omega}=$ $\kappa^{\omega}=\kappa$. Let $\mathscr{B}_{1}$ be the free algebra generated by $\kappa^{+}$generators. Then the free product $\mathscr{B}=\operatorname{Col}\left(\omega_{1}, \kappa\right) \times \mathscr{B}_{1}$ is the desired example. The algebra $\mathscr{B}$ is $\left(\omega_{1}, \cdot, \kappa\right)$ nowhere distributive since $\operatorname{Col}\left(\omega_{1}, \kappa\right)$ is; $\operatorname{hd}(\mathscr{B})>\kappa$ since $\operatorname{hd}\left(\mathscr{B}_{1}\right)=\kappa^{+}$. Obviously $\mathscr{B}$ has no $\omega_{1}$-closed dense subset.

The system $\tilde{F}=\{\langle f, \mathbf{1}\rangle: f \in F\}$ is a system of cardinality $\kappa$ having no disjoint refinement. Suppose the contrary. Let $D$ be a disjoint refinement of $\tilde{F}$. Without loss of generality we may assume that the members of $D$ are of the form $\langle f, x\rangle$ with $f \in F, x \in \mathscr{B}_{1}^{+}$. Choose arbitrary $\left\langle f_{0}, x_{0}\right\rangle \in D$; suppose $\left\langle f_{\alpha}, x_{\alpha}\right\rangle \in D$ have been found for $\alpha<\beta<\omega_{1}$ in such a manner that $f_{0} \varsubsetneqq f_{1} \subsetneq \ldots \varsubsetneqq f_{\alpha} \varsubsetneqq \ldots$ Let $g=\cup\left\{f_{\alpha}: \alpha<\beta\right\} \cup(\gamma, 0)$, where $\gamma \notin \cup\left\{\operatorname{dom}\left(f_{\alpha}\right): \alpha<\beta\right\}$. Then $\langle g, 1\rangle \in \tilde{F}$ and $\langle g, 1\rangle$ contains no $\left\langle f_{\alpha}, x_{\alpha}\right\rangle$ with $\alpha<\beta$. Hence there is some $\left\langle f_{\beta}, x_{\beta}\right\rangle \in D$ with $\langle g, 1\rangle \geqslant\left\langle f_{\beta}, x_{\beta}\right\rangle$. Obviously $f_{\beta} \supsetneq f_{\alpha}$ for all $\alpha<\beta$. We have found a subset $\left\{\left\langle f_{\alpha}, x_{\alpha}\right\rangle: \alpha<\omega_{1}\right\}$ of $D$ such that the $f_{\alpha}$ 's form a nested sequence. Anyway, $D$ is disjoint, hence the set $\left\{x_{\alpha}: \alpha<\omega_{1}\right\} \subseteq \mathscr{B}_{1}^{+}$must be disjoint, too. But this contradicts the well-known fact that hsat $\left(\mathscr{B}_{1}\right)=\omega_{1}$.

2. Refinement properties of centered systems. In the previous section, we have discussed some conditions on $\mathscr{B}$ which guarantee the existence of a disjoint refinement. Now we restrict our attention to special families in $\mathscr{B}$, namely, to families having the finite intersection property.

2.1. Definition. Let $\mathscr{B}$ be a Boolean algebra, $A \subseteq \mathscr{B}$. The set $A$ is centered, or equivalently, $A$ has the finite intersection property (FIP), if $\wedge A^{\prime} \neq 0$ whenever $A^{\prime} \subseteq A$ is finite. A Boolean algebra has a refinement property for centered systems of power at most $\kappa(\mathscr{B}$ has $\operatorname{Rfip}(\kappa))$, if each indexed family $\left\{a_{\alpha}: \alpha<\kappa\right\} \subseteq \mathscr{B}$ with FIP has a disjoint refinement.

It turns out that $\operatorname{Rfip}(\kappa)$ no more holds for $\mathscr{B}$ and $\operatorname{Comp}(\mathscr{B})$ simultaneously, as it was the case of $\operatorname{Rp}(\kappa)$. Indeed, if $\operatorname{Comp}(\mathscr{B})$ has $\operatorname{Rfip}(\kappa)$, then so has $\mathscr{B}$. Nevertheless, $\mathcal{P}(\omega) /$ fin has $\operatorname{Rfip}(c)\left(\left[\mathbf{B V}_{2}\right]\right)$, but $\operatorname{Comp}(\mathscr{P}(\omega) /$ fin $)$ need not satisfy Rfip(c) (2.11). Hence Rfip( $\kappa)$ will be studied mainly for complete Boolean algebras.

Suppose $\kappa$ infinite. When asking whether a complete Boolean algebra $\mathscr{B}$ has $\operatorname{Rfip}(\kappa)$, there is only one interesting case, namely hsat $(\mathscr{B})=\kappa^{+}: \operatorname{Rfip}(\kappa)$ does not hold if $\operatorname{hsat}(\mathscr{B})<\kappa^{+}$and the case $\operatorname{hsat}(\mathscr{B})>\kappa^{+}$is covered by 1.5 . The extremal situation occurs if, in addition, $d(\mathscr{B})=\kappa$ (see $1.12,1.13)$. In what follows we give the solution of the following problem from $\left[\mathbf{B V}_{\mathbf{1}}\right]$ : "Is there a complete Boolean algebra $\mathscr{B}$ which has $\operatorname{Rfip}(\kappa)$ and hsat $(\mathscr{B})=\kappa^{+}$and $d(\mathscr{B})=\kappa$ ?"

We shall show that the answer is affirmative for $\kappa=\omega$ or $\kappa$ singular (of arbitrary cofinality!) in 2.2. The case of regular uncountable $\kappa$ is not decided by the usual axioms of set theory, and we shall show that some simple set-theoretical assumptions (each satisfied by a number of well-known models of ZFC) decide the validity of the statement or of its negation (Theorems 2.3, 2.8). 
The rest of this section is devoted to the consistency proof mentioned in the introduction and to some application of the general results.

2.2. TheOREM. Let $\mathscr{B}$ be a complete Boolean algebra, let $\kappa=\omega$ or a singular cardinal. Then $\mathscr{B}$ has $\operatorname{Rfip}(\kappa)$ if and only if $\operatorname{hsat}(\mathscr{B}) \geqslant \kappa^{+}$.

Proof. The condition hsat $(\mathscr{B}) \geqslant \kappa^{+}$is obviously necessary.

Let $F$ be a centered system in $\Re,|F|=\kappa$. If $\kappa=\omega$, it is an easy exercise to show that $F$ is $\omega$-decomposable, so let $\kappa>\omega$ and $\tau=\operatorname{cf}(\kappa)<\kappa$. Let us assume that each finite meet of members of $F$ belongs to $F$, too.

Claim. $F$ is $\tau^{+}$-decomposable.

For $\xi<\tau$ choose $F_{\xi} \subset F$ such that $\left|F_{\xi}\right|<\kappa, F_{\xi} \subseteq F_{\eta}$ for $\xi<\eta<\tau$ and $\cup\left\{F_{\xi}: \xi<\tau\right\}=F$.

Transfinite recursion. Let $\gamma<\tau$ and suppose that for $\alpha<\beta<\gamma$ we have found ordinals $\xi(\alpha)$ and disjoint systems $C_{\alpha}, C_{\alpha \beta}$ satisfying the following conditions:

(i) $\xi(\alpha)<\xi(\beta)<\tau$ for each $\alpha<\beta<\gamma$;

(ii) if $\alpha<\gamma, x \in F_{\xi(\alpha)}$, then $\left|C_{\alpha}\right|=\left|x \wedge \wedge C_{\alpha}\right|=\tau^{+}$;

(iii) if $\alpha<\beta<\gamma$, then $C_{\alpha \beta} \subseteq C_{\alpha}$ and $\left|C_{\alpha \beta}\right| \leqslant \tau$;

(iv) if $\alpha<\beta<\gamma$, then $\left(C_{\alpha}-C_{\alpha \beta}\right) \cup C_{\beta}$ is a disjoint system.

Define $W_{\gamma}=\cup\left\{C_{\alpha}-\cup\left\{C_{\alpha \beta}: \alpha<\beta<\gamma\right\}: \alpha<\gamma\right\}$. Then $W_{\gamma}$ is disjoint and $\left|W_{\gamma}\right|=\tau^{+}$. If $\left|x \wedge \wedge W_{\gamma}\right|=\tau^{+}$for each $x \in F$, we are done: $F$ is $\tau^{+}$-decomposable.

If there is some $x \in F$ with $\left|x \wedge \wedge W_{\gamma}\right| \leqslant \tau$, then $x \notin F_{\xi(\alpha)}$ for $\alpha<\gamma$ by (ii), hence there is some $\xi(\gamma) \geqslant \sup \{\xi(\alpha): \alpha<\gamma\}, \xi(\gamma)<\tau$, such that $x \in F_{\xi(\gamma)}$.

Define $C_{\alpha \gamma}=\left\{c \in C_{\alpha}: c \wedge x \neq \mathbf{0}\right\}$. Clearly $\left|C_{\alpha \gamma}\right| \leqslant \tau$ for $\alpha<\gamma$.

Since $\left|F_{\xi(\gamma)}\right|^{+}<\kappa<\operatorname{hsat}(\Re)$, there is a disjoint refinement $D$ of the system $\left\{x \wedge y: y \in F_{\xi(\gamma)}\right\}$ by 1.5. For $d \in D$, let $\left\{d_{\iota}: \iota \in \tau^{+}\right\}$be an arbitrary disjoint family in $\mathscr{B} \uparrow d$. Let $C_{\gamma}=\left\{\bigvee\left\{d_{\iota}: d \in D\right\}: \iota<\tau^{+}\right\}$. Clearly $\left|y \wedge \wedge C_{\gamma}\right|=\tau^{+}$for each $y \in F_{\xi(\gamma)}$.

This completes the recursive definitions.

Suppose that the induction has not stopped before $\tau$. We define

$$
C=\cup\left\{C_{\alpha}-\cup\left\{C_{\alpha \beta}: \alpha<\beta<\tau\right\}: \alpha<\tau\right\} .
$$

It is easy to see that $|C|=|x \wedge \wedge C|=\tau^{+}$for each $x \in F$.

Having proved the claim we know that $F$ is $\tau^{+}$-decomposed by $C=\left\{c_{\eta}\right.$ : $\left.\eta \in \tau^{+}\right\}$. For $x \in F$, let $u_{x}=\left\{\eta \in \tau^{+}: x \wedge c_{\eta} \neq \mathbf{0}\right\}$. The family $F$ is closed under finite intersections, so $U=\left\{u_{x}: x \in F\right\}$ is a uniformly centered collection of subsets of $\tau^{+}$. Moreover, $\tau$ is regular. Hence $U$ is $\tau$-decomposable (see, e.g., [Ch], [KP] or [CN, 8.36]). If the pairwise disjoint collection $\left\{a_{\iota}: \iota \in \tau\right\}$ of subsets of $\tau^{+}$ witnesses to the $\tau$-decomposability of $U$, then the system $\left\{\bigvee\left\{c_{\eta}: \eta \in a_{\imath}\right\}: \iota \in \tau\right\}$ witnesses to the $\tau$-decomposability of $F$. Now the theorem follows by 1.6 .

2.3. ThEOREM. Let $\kappa$ be an uncountable cardinal, $\mathscr{B}$ a $\kappa$-complete Boolean algebra with $d(\Re) \leqslant \kappa$ and suppose $\kappa \kappa=\kappa$. Then there is a system consisting of at most $\kappa$ elements of $\mathfrak{B}^{+}$which generates a nontrivial ultrafilter on $\mathscr{B}$. Consequently, $\mathscr{B}$ has not $\operatorname{Rfip}(\kappa)$. 
Before proving the theorem, let us give a definition and an important lemma, which is due to Kunen, van Mill and Mills.

2.4. Definition. Let $\mathscr{B}$ be a Boolean algebra, $\kappa$ an infinite cardinal. A tower $T$ of length $\kappa$ is a family $\left\{t_{\alpha}: \alpha \in \kappa\right\} \subset \mathscr{B}^{+}$satisfying $t_{\alpha} \geqslant t_{\beta}$ whenever $\alpha<\beta<\kappa$. A tower $T$ in $\mathscr{B}$ is called nowhere dense if for each $b \in \mathscr{B}^{+}$there is some $t_{\alpha} \in T$ with $b-t_{\alpha} \neq \mathbf{0}$.

2.5. LEMMA [KvMM]. Let $\kappa$ be an uncountable regular cardinal, let $\mathfrak{B}$ be a Boolean algebra, $|\mathfrak{B}| \leqslant \kappa$. Then there is an ultrafilter on $\mathscr{B}$ containing no nowhere dense tower of length $\kappa$.

Proof. If $|\Re|<\kappa$, the lemma holds trivially since there is no nowhere dense tower of length $\kappa$ in $\mathscr{B}$.

If $|\mathscr{B}|=\kappa$, well-order $\mathscr{B}=\left\{b_{\alpha}: \alpha \in \kappa\right\}$ and define $\mathscr{B}_{\alpha}$ to be the smallest subalgebra of $\mathscr{B}_{3}$ generated by $\left\{b_{\beta}: \beta<\alpha\right\}$. We have $\mathscr{B}_{0} \subseteq \mathscr{B}_{1} \subseteq \ldots \subseteq \mathscr{B}_{\alpha}$ $\subseteq \ldots$, for $\mu<\kappa$ and $\mu$ limit, $\mathscr{B}_{\mu}=\bigcup\left\{\mathscr{B}_{\alpha}: \alpha<\mu\right\}$.

Claim. Let $T$ be a nowhere dense tower of length $\kappa$ in $\mathscr{B}$. Then there is some $\alpha<\kappa$ such that $T \cap \mathscr{B}_{\alpha}$ is a nowhere dense tower in $\mathscr{B}_{\alpha}$.

Let $\gamma<\kappa$ be given. For $b \in \Re_{\gamma}^{+}$, there is some $t \in T$ with $0 \neq b-t$. Denote by $\beta_{b}$ the first $\beta \in \kappa$ such that $t \in \mathscr{B}_{\beta}$ : let $\gamma^{*}=\sup \left\{\beta_{b}: b \in \mathscr{B}_{\gamma}^{+}\right\}$. Since $\kappa$ is regular and $\left|\mathscr{G}_{\gamma}\right|<\kappa, \gamma^{*}<\kappa$, too. Pick $\alpha_{0}<\kappa$ arbitrarily, and define $\alpha_{n+1}=\alpha_{n}^{*}, \alpha=$ $\sup \left\{\alpha_{n}: n \in \omega\right\}$. Since $\kappa$ is regular and uncountable, $\alpha<\kappa$. If $b \in \mathscr{B}_{\alpha}$, then $b \in \mathscr{B}_{\alpha_{n}}$ for some $\alpha_{n}<\omega$ and there is a $t \in T \cap \mathscr{S}_{\alpha_{n+1}}$ with $b-t \neq \mathbf{0}$. Thus $T \cap \mathscr{B}_{\alpha}$ is nowhere dense in $\mathscr{H}_{\alpha}$.

For a nowhere dense tower $T$ of length $\kappa$ in $\mathscr{B}$, let $\alpha(T)<\kappa$ be such that $T \cap \mathscr{B}_{\alpha(T)}$ is nowhere dense in $\mathscr{B}_{\alpha(T)}$ and let $t(T) \in T$ be such that $t(T) \leqslant t$ for each $t \in T \cap \mathscr{B}_{\alpha(T)}$.

The family $F=\{1-t(T): T$ is a nowhere dense tower of length $\kappa$ in $\mathscr{B}\}$ is centered: Pick $n \in \omega, T_{1}, T_{2}, \ldots, T_{n}$ nowhere dense towers of length $\kappa$ in $\mathscr{B}$. Let $\mathscr{B}^{i}=\mathscr{H}_{\alpha\left(T_{i}\right)}, i=1, \ldots, n$, and suppose $\mathscr{B}^{1} \subseteq \mathscr{B}^{2} \subseteq \ldots \subseteq \mathscr{B}^{n}$. The tower $T_{1} \cap$ $\mathscr{B}^{1}$ is nowhere dense in $\mathscr{B}^{1}$, thus there is $t^{1} \in T_{1} \cap \mathscr{B}^{1}, 1-t^{1} \neq 0$. But $1-t^{1} \in$ $\mathscr{B}^{1} \subseteq \mathscr{B}^{2}$ and $T_{2} \cap \mathscr{B}^{2}$ is nowhere dense in $\mathscr{B}^{2}$, hence there is some $t^{2} \in T_{2} \cap \mathscr{B}^{2}$ with $\left(1-t^{1}\right)-t^{2} \neq 0$; proceeding further, we shall find $t^{3}, t^{4}, \ldots, t^{n}$ such that $1-\left(t^{1} \vee t^{2} \vee \ldots \vee t^{n}\right) \neq 0$.

As $t\left(T_{i}\right) \leqslant t^{i}$, we obtain

$$
\mathbf{0} \neq \wedge\left\{1-t^{i}: i=1, \ldots, n\right\} \leqslant \wedge\left\{1-t\left(T_{i}\right): i=1, \ldots, n\right\} .
$$

Obviously, if $U$ is an ultrafilter on $\mathscr{B}$ and $U \supseteq F$, then $U$ contains no nowhere dense tower of length $\kappa$ in $\mathscr{B}$.

Proof of Theorem 2.3. Let $C$ be a dense subset of $\mathscr{B}$ of cardinality at most $\kappa$. Denote by $\mathscr{D}$ the smallest subalgebra of $\operatorname{Comp}(\mathscr{B})$ that contains $C$ as well as all joins $\bigvee C^{\prime}$, where $C^{\prime} \in[C]^{<\kappa}$. Then $\mathscr{D} \subseteq \mathscr{B}$, since $\mathscr{B}$ is $\kappa$-complete, and $|\mathscr{D}|=$ $|\mathcal{C}|^{\kappa} \leqslant \kappa^{\kappa}=\kappa$; thus by 2.5 , there is some ultrafilter $U$ on $\mathscr{D}$ containing no nowhere dense tower of length $\kappa$ in $\mathscr{B}$. Clearly $|U| \leqslant|\mathscr{D}| \leqslant \kappa$ and $U \subseteq \mathscr{B}$.

We shall show that $U$ generates an ultrafilter on $\mathscr{B}$. Let $x, y \in \mathscr{B}^{+}$be such that $x \wedge y=0, x \vee y=1$. Let $Z \subseteq C$ be a maximal disjoint family refining $\{x, y\}$. If 
$|Z|<\kappa$, then $x=\bigvee\{c \in Z: c \leqslant x\}$ belongs to $\mathscr{D}$ as well as $y$ does, thus either $x$ or $y$ belongs to $U$. If $|Z|=\kappa$, say $Z=\left\{c_{\alpha}: \alpha \in \kappa\right\}$, then there is some $\mu<\kappa$ such that $\bigvee\left\{c_{\alpha}: \alpha<\mu\right\} \in U$. In the opposite case each join $\bigvee\left\{c_{\alpha}: \mu \leqslant \alpha<\kappa\right\}$ belongs to $U$, but the family of all such joins is a nowhere dense tower of length $\kappa$ in $\mathscr{D}$, which contradicts our choice of $U$. Now either $\bigvee\left\{c_{\alpha}: \alpha<\mu \& c_{\alpha} \leqslant x\right\}$ or $\bigvee\left\{c_{\alpha}: \alpha<\mu \& c_{\alpha} \leqslant y\right.$. $\}$ belongs to $U$, hence $U$ generates an ultrafilter on $\mathscr{B}$.

Finally, assume that, on the contrary, $\mathscr{B}$ has $\operatorname{Rfip}(\kappa)$. Then hsat $(\mathscr{B})=\operatorname{hsat}(\mathscr{D})$ $=\kappa^{+}$; hence for the centered system $U$ described above we have $\mid\{v<u$; $v \in U\} \mid=\kappa$ for each $u \in U$. Let $D$ be a disjoint refinement of $U, D \subseteq C$. Pick some maximal disjoint family $Z \subseteq C$ extending $D$. Let $Z=\left\{z_{\alpha}: \alpha<\kappa\right\}$ be a one-one numbering of $Z$. Define $t_{\alpha}=\bigvee\left\{z_{\beta}: \alpha \leqslant \beta<\kappa\right\}, T=\left\{t_{\alpha}: \alpha<\kappa\right\}$. Then $T \subseteq \mathcal{D}, T$ is a nowhere dense tower of length $\kappa$, hence $T \& U$. Anyway, the family $T \cup U$ is centered and $U$ is an ultrafilter on $\mathscr{Q}$, thus $T \subseteq U$-a contradiction.

\subsection{Corollary. Assume $(\mathrm{CH})$. Then $\operatorname{Col}\left(\omega, \omega_{1}\right)$ has not $\mathrm{Rfip}\left(\omega_{1}\right)$.}

2.7. Definition. Let $\lambda$ be an infinite cardinal. The formula $f \leqslant g$ iff $\mid\{\alpha \in \lambda$ : $f(\alpha)>g(\alpha)\} \mid<\lambda$ defines an order on ${ }^{\lambda} \lambda$. A family $F \subset{ }^{\lambda} \lambda$ is dominating, if for every $g \in{ }^{\lambda} \lambda$ there is some $f \in F$ with $f \geqslant g$. If $F$ is a dominating family in ${ }^{\lambda} \lambda$, $|F|=\mu$ and the order $\leqslant$ on $F$ is of the type $\mu$, then $F$ is called a $\mu$-scale.

2.8. TheOREM. Let $\lambda \leqslant \kappa$ be infinite cardinals. Let $\mathscr{B}$ be $a(\lambda, \cdot, \kappa)$-nowhere distributive Boolean algebra which is $(\tau, \cdot, 2)$-distributive for each $\tau<\lambda$. Let $A=$ $\left\{a_{\xi}: \xi<\kappa\right\} \subseteq \Re^{+}$be $\lambda$-decomposable. Then $A$ has a disjoint refinement provided there is no dominating family of power $\kappa$ in ${ }^{\lambda} \lambda$.

Proof. Let $P=\{p(\alpha): \alpha \in \lambda\} \subset \mathscr{B}^{+}$be the disjoint set witnessing to $\lambda$-decomposability of $A$. For each $\alpha<\lambda$, there is a matrix $2(\alpha)=\{Q(\alpha, \beta): \beta \in \lambda\}$ in $\mathscr{B}\lceil p(\alpha)$ satisfying 1.14(iii), (v). (Notice that the proof of these statements required the $(\tau, \cdot, 2)$-distributivity only, $\tau<\lambda$. Analogously, $\lambda$ is regular.)

We shall construct a mapping $f_{\xi} \in{ }^{\lambda} \lambda$ for $\xi \in \kappa$ recursively as follows:

Suppose $f_{\xi}(\beta)$ has been defined for $\beta<\alpha$. Let $\tilde{\alpha} \geqslant \alpha$ be the first ordinal number such that $a_{\xi} \wedge p(\tilde{\alpha}) \neq \mathbf{0}$. Let $\gamma \in \lambda$ be the smallest ordinal with $\gamma \geqslant f_{\xi}(\beta)$ for $\beta<\alpha$ and $\left|a_{\xi} \wedge \wedge Q(\tilde{\alpha}, \gamma)\right| \geqslant \kappa$. Define $f_{\xi}(\alpha)=\gamma$.

The family $\left\{f_{\xi}: \xi \in \kappa\right\} \subset{ }^{\lambda} \lambda$ is not dominating in ${ }^{\lambda} \lambda$. Thus there is some $g \in{ }^{\lambda} \lambda$ satisfying $\left\{\alpha \in \lambda: g(\alpha)>f_{\xi}(\alpha)\right\} \mid=\lambda$ for each $\xi \in \kappa$. According to the regularity of $\lambda$ we may assume that $g$ is strictly increasing. Let us define

$$
Q=\cup\{Q(\alpha, g(\alpha)): \alpha \in \lambda\} .
$$

Claim. For each $\xi \in \kappa,\left|a_{\xi} \wedge \wedge Q\right| \geqslant \kappa$.

Fix $\xi \in \kappa$, let $X_{\xi}=\left\{\alpha \in \lambda: g(\alpha)>f_{\xi}(\alpha)\right\}, \quad Y_{\xi}=\left\{\alpha \in \lambda: a_{\xi} \wedge p(\alpha)\right\} \neq \mathbf{0}$. If $\alpha \in X_{\xi} \cap Y_{\xi}$, then $\left|a_{\xi} \wedge \wedge Q\left(\alpha, f_{\xi}(\alpha)\right)\right| \geqslant \kappa$, thus $\left|a_{\xi} \wedge \wedge Q\right| \geqslant \kappa$. Thus it suffices to show that $X_{\xi} \cap Y_{\xi} \neq \varnothing$. Choose an arbitary $\alpha \in X_{\xi}$. By our construction of $f_{\xi}$, $f_{\xi}(\alpha)=f_{\xi}(\tilde{\alpha})$, where $\tilde{\alpha}$ was defined in the recursive procedure. The function $g$ is increasing, thus we have $g(\tilde{\alpha}) \geqslant g(\alpha)>f_{\xi}(\alpha)=f_{\xi}(\tilde{\alpha})$, so $\tilde{\alpha} \in X_{\xi}$. Anyway, $\tilde{\alpha} \in Y_{\xi}$.

Having proved the claim, it remains to apply 1.2 in order to obtain a disjoint refinement of $A$. 
2.9. Corollary. Let $\mathscr{B}$ be an $\left(\omega, \cdot, \omega_{1}\right)$-nowhere distributive Boolean algebra, let $A \subset \mathscr{B}^{+}$have FIP and $|A| \leqslant \omega_{1}$. If there is no dominating family of power $\omega_{1}$ in ${ }^{\omega} \omega$, then $A$ has a disjoint refinement.

Proof. Let $U$ be an arbitrary ultrafilter on $\mathscr{B}, U \supseteq A$. Let $W$ be a maximal disjoint system in $\mathscr{B}^{+}$such that for each $w \in W, 1-w \in U$. Clearly $a \wedge \wedge W$ is infinite for each $a \in A$. If there is some $a_{0} \in A$ such that $\left|a_{0} \wedge \wedge W\right|=\omega$, let $P=\left\{w \in W: a_{0} \wedge w \neq 0\right\}$. Since $A$ has FIP, $|a \wedge \wedge P|=\omega$ for each $a \in A$, hence 2.8 may be applied. If $|a \wedge \wedge W|=\omega_{1}$ for each $a \in A$, then the disjoint refinement of $A$ exists by 1.2 .

2.10. Corollary. Assume there is no $\omega_{1}$-scale in ${ }^{\omega} \omega$. Then $\operatorname{Col}\left(\omega, \omega_{1}\right)$ has $\operatorname{Rfip}\left(\omega_{1}\right)$.

APPLICATIONS. I. The algebra $\operatorname{Comp}(\mathscr{P}(\omega) /$ fin $)$ and the property $\operatorname{Rfip}\left(2^{\omega}\right)$.

The following negative solution is a direct consequence of Theorem 2.3.

2.11. Proposition. Suppose $c^{c}=c$. Then there is an ultrafilter on $\operatorname{Comp}(\mathscr{P}(\omega) /$ fin $)$ generated by at most $\mathrm{c}$ elements. Hence under $\mathfrak{c}^{\mathfrak{c}}=\mathrm{c}$, the algebra $\operatorname{Comp}(\mathscr{P}(\omega) / \mathrm{fin})$ has not Rfip(c).

A result similar to the proposition above as well as Corollary 2.6 was obtained independently by $\mathbf{R}$. Laver under the assumption $\diamond\left(\omega_{1}\right)[\mathbf{L}]$.

The set-theoretical assumption cannot be omitted from 2.11. The forthcoming corollary of Theorem 2.2 shows the positive answer:

2.12. Proposition. If $2^{\omega}$ is a singular cardinal, then the complete Boolean algebra $\operatorname{Comp}\left(\mathscr{P}(\omega) /\right.$ fin) has $\operatorname{Rfip}\left(2^{\omega}\right)$. Therefore no ultrafilter on $\operatorname{Comp}(\mathscr{P}(\omega) /$ fin $)$ is generated by $\leqslant 2^{\omega}$ elements.

It is worth mentioning that in this case of $2^{\omega}$ being singular we obtain an alternative proof of the fact that $\mathscr{P}(\omega) /$ fin satisfies $\operatorname{Rfip}\left(2^{\omega}\right)\left(\operatorname{see}\left[\mathbf{B V}_{2}\right]\right)$.

Now we shall describe a model $\Re$ of a set theory in which $\operatorname{Comp}(\mathcal{P}(\omega) /$ fin) also has $\operatorname{Rfip}\left(2^{\omega}\right)$ and, moreover, $2^{\omega}$ is a regular cardinal.

Let us start with a formulation of combinatorial assumptions, which imply that $\operatorname{Rfip}\left(2^{\omega}\right)$ holds for $\operatorname{Comp}(\mathscr{P}(\omega) /$ fin $)$.

2.13. Proposition. Suppose that $2^{\omega}=\omega_{2}$ and

(i) $\operatorname{Comp}(\mathscr{P}(\omega) /$ fin $)$ is isomorphic to $\operatorname{Col}\left(\omega_{1}, \omega_{2}\right)$,

(ii) each ultrafilter on $\mathscr{P}(\omega) /$ fin contains a nowhere dense tower in $\mathscr{P}(\omega) / \mathrm{fin}$; and

(iii) there is no $\omega_{2}$-scale in ${ }^{\omega_{1}} \omega_{1}$.

Then $2^{\omega_{1}}>\omega_{2}$ and $\operatorname{Comp}(\mathscr{\rho}(\omega) /$ fin $)$ has $\operatorname{Rfip}\left(2^{\omega}\right)$. Therefore there is no ultrafilter on $\operatorname{Comp}(\mathscr{P}(\omega) /$ fin $)$ having $\leqslant 2^{\omega}$ generators.

Proof. The fact $2^{\omega_{1}}>\omega_{2}$ follows from (iii). Denote $\mathscr{B}=\operatorname{Comp}(\mathscr{P}(\omega) /$ fin), let $A=\left\{a_{\alpha}: \alpha \in \omega_{2}\right\}$ be a centered family of elements of $\mathscr{B}$. Then there is an ultrafilter $U$ on $\mathscr{P}(\omega) /$ fin which is compatible with $A$. Let $T$ be a nowhere dense tower on $\mathcal{P}(\omega) /$ fin, $T \subseteq U$. If the length of $T$ is $\omega_{2}$, then $A$ is $\omega_{2}$-decomposable and 
hence $A$ has a disjoint refinement by 1.2. If the length of $T$ equals to $\omega_{1}$, then $A$ is $\omega_{1}$-decomposable; now by (i) and (iii) there is a disjoint refinement of $A$ by Theorem 2.8.

2.14. The model. We shall describe the basic situation without discussing possible generalizations.

Let $\Re$ be a countable transitive model of $\mathrm{ZFC}+\mathrm{GCH}$. Let $\mathscr{B}_{0}, \mathscr{B}_{1}$, $\mathscr{B}_{2}$ be the complete Boolean algebras in $\mathscr{N}$ such that

$\mathscr{G}_{0}$ is the Solovay-Tennenbaum algebra making $M A+2^{\omega}=\omega_{2}$;

$\mathscr{B}_{1}$ is the well-known algebra for adding $\omega_{1}$ Cohen reals;

$\mathscr{B}_{2}$ is the algebra with the basis $\left\{f: \operatorname{dom}(f) \rightarrow \omega_{1}: \operatorname{dom}(f) \in\left[\omega_{3}\right]^{<\omega_{1}}\right\}$ ordered by the inverse inclusion (observe that $\mathscr{B}_{2}$ is isomorphic to the algebra with the base $\left.\left\{f: \operatorname{dom}(f) \rightarrow\{0,1\}: \operatorname{dom}(f) \in\left[\omega_{3}\right]^{<\omega_{1}}\right\}\right)$.

Let $\mathscr{B}=\mathscr{B}_{0} \times \mathscr{B}_{1} \times \mathscr{B}_{2}$ be the free product, and $G$ be generic ultrafilter on $\mathscr{B}$. Denote by $G_{1}$, resp. $G_{2}$, the restriction of $G$ to $\mathscr{B}_{0} \times \mathscr{B}_{1}$, resp. to $\mathscr{B}_{2}$. The algebra $\mathscr{B}_{0} \times \mathscr{B}_{1}$ satisfies c.c.c.; the algebra $\mathscr{B}_{2}$ has an $\omega_{1}$-closed basis, hence by the Easton-type argument $\mathscr{P}(\omega)^{\mathscr{T} U G]}=\mathscr{P}(\omega)^{\mathscr{T}\left[G_{1}\right]}$ and in $\mathscr{N}[G]$ all cardinals and cofinalities are preserved.

As shown in [BFM], $\Re\left[G_{1}\right] \vDash 2^{\omega}=\omega_{2}$ and there are two increasing families $\left\{f_{\alpha}\right.$ : $\left.\alpha \in \omega_{1}\right\},\left\{g_{\beta}: \beta \in \omega_{2}\right\}$ in ${ }^{\omega} \omega$ without an upper bound.

The same holds in $\mathfrak{N}[G]$. By [BPS], the existence of a family $\left\{f_{\alpha}: \alpha<\omega_{1}\right\}$ $\subseteq{ }^{\omega} \omega$ without an upper bound implies $\operatorname{Col}\left(\omega_{1}, 2^{\omega}\right) \cong \operatorname{Comp}(\mathcal{P}(\omega) /$ fin $)$. By [BFM], if there are two increasing families $\left\{f_{\alpha}: \alpha \in \omega_{1}\right\}$ and $\left\{g_{\beta}: \beta \in \omega_{2}\right\}$ in ${ }^{\omega} \omega$ without an upper bound, then each ultrafilter on $\mathscr{P}(\omega)$ /fin contains a nowhere dense tower. Moreover, $\mathscr{R}[G] \vDash 2^{\omega_{1}}=\omega_{3}$ and each dominating family in ${ }^{\omega_{1}} \omega_{1}$ is of cardinality $\omega_{3}$. Thus all the assumptions of Proposition 2.13 hold in $\mathscr{T}[G]$ and we conclude

$$
\mathscr{M}[G] \vDash 2^{\omega}=\omega_{2} \& \operatorname{Comp}(\mathscr{P}(\omega) / \text { fin }) \text { has } \operatorname{Rfip}\left(2^{\omega}\right) \text {. }
$$

II. Fodor's conjecture. Fodor's conjecture states that $\mathscr{P}\left(\omega_{1}\right) / N S\left(\omega_{1}\right)$ has $\operatorname{Rp}\left(\omega_{1}\right)$, where $N S\left(\omega_{1}\right)$ denotes the ideal of all nonstationary subsets of $\omega_{1}$ [BHM]. In what follows let $\mathscr{P}=\mathscr{P}\left(\omega_{1}\right) / i$, where $i$ is an arbitrary $\omega_{1}$-complete ideal on $\mathscr{P}\left(\omega_{1}\right)$ containing $i_{F}$. The algebra $\mathscr{B}$ is always $\left(\omega, \cdot, \omega_{1}\right)$-nowhere distributive (Ulam [U]); thus hsat $(\mathscr{B}) \geqslant \omega_{2}$. A. Taylor $[\mathbf{T}]$ and Balcar and Vojtáš $\left[\mathbf{B V}_{\mathbf{1}}\right]$ proved independently that $\mathscr{B}$ has not $\operatorname{Rp}\left(\omega_{1}\right)$ if and only if there is some $a \in \mathscr{P}\left(\omega_{1}\right)-i$ such that $\mathscr{B} \uparrow[a]$ is isomorphic to $\operatorname{Col}\left(\omega, \omega_{1}\right)$ or, equivalently, $\mathscr{B} \uparrow[a]$ has a dense subset of size $\omega_{1}$. ([a] denotes the element of $\mathscr{B}$ represented by $a \subseteq \omega_{1}$.)

Let us complete the general theorems 1.5, 1.12, 2.3 and 2.8 by the following ones, which deal with this particular $\mathscr{B}$.

2.15. Proposition. Suppose $(\mathrm{CH})$ and let $i$ be a nontrivial $\omega_{1}$-complete ideal on $\omega_{1}$, $\mathscr{B}=\mathscr{P}\left(\omega_{1}\right) / i$. Then the following statements are equivalent:

(i) Each family $A \subseteq \mathscr{B}^{+},|A|=\omega_{1}$, has a disjoint refinement;

(ii) each family $A \subseteq \mathscr{B}^{+}$with $F I P,|A|=\omega_{1}$, has a disjoint refinement;

(iii) there is no family $\left\{x_{\alpha}: \alpha<\omega_{1}\right\} \subseteq \mathscr{P}\left(\omega_{1}\right)$ such that the ideal generated by $i \cup\left\{x_{\alpha}: \alpha<\omega_{1}\right\}$ is maximal. 
Proof. (i) $\rightarrow$ (ii) is trivial.

(ii) $\rightarrow$ (iii). It suffices to prove that no ultrafilter on $\mathscr{B}$ has $\omega_{1}$ generators. Let $S=\left\{\left[v_{\alpha}\right]: \alpha<\omega_{1}\right\}$ be a base of some ultrafilter $U$ on $\mathscr{B}$, where $v_{\alpha} \in \mathscr{P}\left(\omega_{1}\right)-i$. Let $\left\{\left[w_{\alpha}\right]: \alpha<\omega_{1}\right\}$ be a disjoint refinement of $S$. Since $i$ is $\omega_{1}$-complete we may assume that $w_{\alpha}$ 's are pairwise disjoint. The algebra $\mathscr{B}$ is atomless; we may split each $w_{\alpha}$ into two disjoint parts $w_{\alpha}(0), w_{\alpha}(1)$ not belonging to $i$. Necessarily, one of the elements $\left[\omega_{1}-\cup\left\{w_{\alpha}: \alpha<\omega_{1}\right\}\right],\left[\cup\left\{w_{\alpha}(0): \alpha<\omega_{1}\right\}\right],\left[\cup\left\{w_{\alpha}(1): \alpha<\omega_{1}\right\}\right]$ belongs to $U$, which contradicts the assumption that $S$ is a base of $U$.

$\neg$ (i) $\rightarrow \neg$ (iii). If (i) does not hold, then there is some $a \in \mathscr{P}\left(\omega_{1}\right)-i$ such that $\mathscr{P}\left(\omega_{1}\right) / i(a)$ has a dense subset of size $\omega_{1}$, where $i(a)=\left\{x \in \mathscr{P}\left(\omega_{1}\right): x \cap a \in i\right\}$, by a result of $[\mathbf{T}]$ or $\left[\mathbf{B V}_{1}\right]$, see also 1.12. Since we assume $2^{\omega}=\omega_{1}$ (this is the only place where we need $\mathrm{CH})$, there is an ultrafilter $U$ on $\mathscr{P}\left(\omega_{1}\right) / i(a)$ generated by $\left\{\left[x_{\alpha}\right]: \alpha<\omega_{1}\right\}$ according to Theorem 2.3. Then, however, the ideal generated by $i \cup\left\{\omega_{1}-a\right\} \cup\left\{\omega_{1}-x_{\alpha}: \alpha<\omega_{1}\right\}$ is maximal.

2.16. Corollary. Suppose $(C H)$. There exists a family $\left\{a_{\alpha}: \alpha<\omega_{1}\right\}$ of stationary subsets of $\omega_{1}$ having no disjoint refinement by stationary subsets if and only if there is a family $\left\{x_{\alpha}: \alpha<\omega_{1}\right\} \subseteq \mathscr{P}\left(\omega_{1}\right)$ such that all closed unbounded subsets of $\omega_{1}$ together with $\left\{x_{\alpha}: \alpha<\omega_{1}\right\}$ generate a uniform ultrafilter on $\omega_{1}$.

2.17. Proposition. If there is no dominating family of power $\omega_{1}$ in ${ }^{\omega} \omega$, then each family $A,|A|=\omega_{1}$ or stationary subsets of $\omega_{1}$ closed under finite intersections has a disjoint refinement consisting of stationary sets.

Proof. Apply 2.9.

2.18. REMARKS. Let $\mathscr{B}$ be $\mathscr{P}\left(\omega_{1}\right)$ modulo an $\omega_{1}$-complete ideal $i, i \supseteq i_{F}$.

(a) It is consistent that hsat $(\mathscr{B})>\omega_{2}$ (for all $i$ 's); hence $\operatorname{Rp}\left(\omega_{1}\right)$ holds by 1.5 . This follows, e.g., from the weak Kurepa hypotheses [BK], [BHM].

(b) Assuming the existence of a huge cardinal, Kunen showed the consistency of the existence of an $i$ such that hsat $(\mathscr{B})=\omega_{2}\left[K_{\mathbf{u}_{1}}\right]$.

(c) R. Laver (unpublished result) proved the relative consistency of "there is a nontrivial $\omega_{1}$-complete ideal $i$ on $\omega_{1}$ such that hsat $(\mathscr{B})=\omega_{2} \&$ there is no $\omega_{1}$-scale in ${ }^{\omega} \omega$ ". This is the case when $\operatorname{Rfip}\left(\omega_{1}\right)$ holds by 2.17 .

(d) By 2.15 , if there is an ideal $i$ such that $\operatorname{hsat}(\mathscr{B})=\omega_{2}$ and $d(\mathscr{B})=\omega_{1}$ and $2^{\omega}=\omega_{1}$, then $\operatorname{Rp}\left(\omega_{1}\right)$ as well as $\operatorname{Rfip}\left(\omega_{1}\right)$ does not hold for $\Re$. A consistent example of such an ideal was exhibited by Woodin [W].

(e) It is an open question whether an analogous situation as in (d) may arise for $i=N S\left(\omega_{1}\right)$. The only known model where $\operatorname{hsat}\left(\mathcal{P}\left(\omega_{1}\right) / N S\left(\omega_{1}\right)\right)=\omega_{2}$ holds was constructed by Van Wesep [VW]. In his model, $2^{\omega}=\omega_{2}$.

3. Extensions of filters. Having shown that there is a close connection between the disjoint refinement property for centered systems and extension of filters to ultrafilters, let us now consider the following problem: Given a Boolean algebra $\mathscr{B}$ and a filter $\mathscr{F}$ on $\mathscr{B}$, what is the least cardinality of a subset $\mathcal{C} \subset \mathscr{B}$ such that $\mathscr{F} \cup \mathcal{C}$ generates an ultrafilter on $\mathscr{B}$ ? Our attention will concentrate on $\mathscr{B}=$ $\mathcal{P}(\kappa) / i_{F}$, where $\kappa$ is an infinite cardinal. 
For convenience, topological terminology will frequently be used in this section.

We shall show by the way that there is a discrete set $D \subset \beta N-N,|D|=\omega_{1}$, with precisely one complete accumulation point. This answers a question posed by R. G. Woods [Wo], van Douwen [vD ], Hušek [Hu] and others. It should be remarked that the same result was obtained independently by $K$. Kunen $\left[\mathbf{K} \mathbf{u}_{2}\right]$ and S. Shelah [S].

3.1. Definition. Let $\mathscr{F}$ be a filter on a Boolean algebra $\mathscr{B}$. The relative character of an ultrafilter $\mathscr{U} \supseteq \mathcal{F}$ with respect to $\mathscr{F}$ is defined by

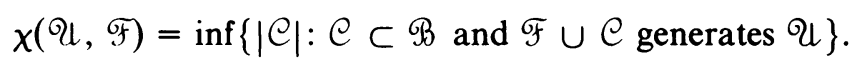

3.2. Definition. Let $\kappa$ be a cardinal. Denote by $\delta$ the family of all functions $\varphi$ such that $\operatorname{dom}(\varphi)$ is a finite subset of $\kappa, \operatorname{rng}(\varphi) \subset\{0,1\}$. For $\varphi \in \mathcal{S}$, the canonical open set in the space ${ }^{\star} 2$ determined by $\varphi$ is the set $H(\varphi)=\left\{f \in{ }^{\star} 2: f \supseteq \varphi\right\}$.

Suppose $U$ to be a nonvoid open subset of ${ }^{\star} 2$. Let

$$
\begin{aligned}
\mathcal{Q}(U)= & \left\{\bigcup\left\{\operatorname{dom}(\varphi): \varphi \in \mathcal{S}^{\prime}\right\}: \mathcal{S}^{\prime} \in[\mathcal{S}]^{<\omega_{1}}\right. \text { and } \\
& \left.\bigcup\left\{H(\varphi): \varphi \in \mathcal{S}^{\prime}\right\} \text { is a dense subset of } U\right\} .
\end{aligned}
$$

3.3. Lemma. Let $U$ be a nonempty open subset of the space ${ }^{*} 2$. Then for each $A \in \mathbb{Q}(U), H(\varphi) \cap U \neq \varnothing$ whenever $\varphi \in \mathcal{S}$ is such that $\operatorname{dom}(\varphi) \cap A=\varnothing$.

Moreover, if $U, V \in{ }^{\star} 2$ are open sets with nonvoid intersection, then for every $A \in \mathbb{Q}(U), B \in \mathbb{Q}(V)$ we have $A \cup B \in \mathbb{Q}(U \cap V)$.

Proof. Let us notice that for each nonvoid open $U \subset 2^{\kappa}$, the set $\mathscr{Q}(U)$ contains at least one nonvoid member. This follows from the well-known fact that each family of pairwise disjoint nonempty open subsets of ${ }^{*} 2$ is at most countable.

Let $A \in \mathbb{Q}(U), B \in \mathbb{Q}(V)$,

$$
A=\cup\left\{\operatorname{dom}(\psi): \psi \in \mathcal{S}^{\prime}\right\}, \quad B=\cup\left\{\operatorname{dom}(\vartheta): \vartheta \in \mathcal{S}^{\prime \prime}\right\}
$$

Suppose $\varphi \in \mathcal{S}, \operatorname{dom}(\varphi) \cap A=\varnothing$. Then for each $\psi \in \mathcal{S}^{\prime}$ the intersection $\operatorname{dom}(\psi) \cap \operatorname{dom}(\varphi)$ is empty. Thus an arbitrary $f \in{ }^{\kappa} 2$ with $f \supset \varphi \cup \psi$ belongs to $H(\varphi) \cap H(\psi)$, consequently to $H(\varphi) \cap U$.

Moreover, suppose $U \cap V \neq \varnothing$. Let $\mathcal{S}^{\prime \prime \prime}=\left\{\psi \cup \vartheta: \psi \in \mathcal{S}^{\prime}, \boldsymbol{\vartheta} \in \mathcal{S}^{\prime \prime}\right.$ and $H(\psi)$ $\cap H(\vartheta) \neq \varnothing\}$. Then $A \cup B \supseteq \cup\left\{\operatorname{dom}(\varphi): \varphi \in \mathcal{S}^{\prime \prime \prime}\right\}$. We have to show that $\cup\left\{\operatorname{dom}(\varphi): \varphi \in \mathcal{S}^{\prime \prime \prime}\right\} \in \mathbb{Q}(U \cap V)$. However, $H(\psi \cup \vartheta)=H(\psi) \cap H(\vartheta) \subset U$ $\cap V$ whenever $H(\psi) \cap H(\vartheta) \neq \varnothing$, and the density of $\cup\left\{H(\varphi): \varphi \in \mathcal{S}^{\prime \prime \prime}\right\}$ in $U \cap V$ follows from the density of sets $\cup\left\{H(\psi): \psi \in \mathcal{\delta}^{\prime}\right\}$ in $U$ and $\cup\left\{H(\vartheta): \vartheta \in \mathcal{S}^{\prime \prime}\right\}$ in $V$, respectively. Since $A \cup B$ is countable and contains a member of $\mathbb{Q}(U \cap V)$, it belongs to $\mathbb{Q}(U \cap V)$, too.

3.4. Lemma. Let $\mu, \nu$ be infinite cardinals, $\nu$ regular, let $\omega_{1} \leqslant \nu \leqslant \mu$. Let $p \in$ $G\left({ }^{\mu} 2\right)$. Then there exists a discrete set $\left\{p_{\xi}: \xi \in \nu\right\} \subset G\left({ }^{\mu} 2\right)-\{p\}$ with the following properties:

(i) for each neighborhood $U$ of $p$ there is some $\alpha<\nu$ such that $U \supset\left\{p_{\xi}\right.$ : $\alpha<\xi<\nu\}$;

(ii) for each $\alpha<\nu, p \notin \operatorname{cl}\left(p_{\xi}: \xi<\alpha\right)$,

(iii) if $q \neq p, q \in G\left({ }^{\mu} 2\right)$, then there is some $\alpha<\nu$ such that $q \notin \operatorname{cl}\left(p_{\xi}: \alpha<\xi<\nu\right)$. 
Proof. Identify $p$ with some ultrafilter $\bigcup_{p}$ in $R O\left({ }^{\mu} 2\right)$. Let $y \in{ }^{\mu} 2$ be the limit point of the ultrafilter $\mathcal{U}_{p}$ in ${ }^{\mu} 2$.

Consider ${ }^{\mu}$ 2. For every ordinal $\xi<\nu$, define $y_{\xi} \in{ }^{\mu} 2$ by the rule $y_{\xi}(\alpha)=1-$ $y(\alpha)$ if $\xi<\alpha<\nu, y_{\xi}(\alpha)=y(\alpha)$ otherwise.

The following facts are almost obvious:

(a) $\left\{y_{\xi}: \xi<\nu\right\}$ is a discrete subspace of ${ }^{\mu} 2$;

(b) for each neighborhood $U$ of $y$ in ${ }^{\mu} 2$, there is some $\alpha<\nu$ such that $U \supset\left\{y_{\xi}\right.$ : $\alpha<\xi<\nu\}$

(c) for each $\alpha<\nu$, there is a neighborhood $U$ of $y$ in ${ }^{\mu} 2$ such that $U \cap\left\{y_{\xi}\right.$ : $\xi<\alpha\}=\varnothing$.

Define $\mathscr{F}_{\xi}=\left\{U \in \mathcal{U}_{p}\right.$ : there is some $A \in \mathbb{Q}(U)$ such that $\left.A \cap \nu \subset \xi\right\}$.

Claim. If $\vartheta_{\xi}$ is a canonical neighborhood system of $y_{\xi}$ in ${ }^{\mu} 2$, then $\vartheta_{\xi} \cup \mathscr{F}_{\xi}$ is a centered system of regular open sets.

Each member of $\mathcal{O}_{\xi} \cup \mathscr{F}_{\xi}$ is regular open. If $U_{1}, U_{2}, \ldots, U_{n} \in \mathscr{F}_{\xi}$, then $U_{1}$ $\cap \ldots \cap U_{n} \in \mathscr{F}_{\xi}$ by Lemma 3.3 and by the definition of $\mathscr{F}_{\xi}$.

Let $H(\varphi) \in \mathcal{O}_{\xi}, U \in \mathscr{F}_{\xi}$. Denote $\varphi_{0}=\varphi \uparrow(\nu-(\xi+1)), \varphi_{1}=\varphi-\varphi_{0}$. Then $H\left(\varphi_{1}\right)$ is a neighborhood of $y$, hence $H\left(\varphi_{1}\right) \cap U$ is a nonvoid regular open set. Fix an $A \in \mathbb{Q}(U)$ such that $A \cap \nu \subset \xi$. Then $\left(A \cup \operatorname{dom}\left(\varphi_{1}\right)\right) \cap \operatorname{dom}\left(\varphi_{0}\right)=\varnothing$; therefore the set $H\left(\varphi_{0}\right) \cap H\left(\varphi_{1}\right) \cap U$ is nonvoid by Lemma 3.3. Since $H\left(\varphi_{0}\right) \cap H\left(\varphi_{1}\right)$ $=H(\varphi)$, the claim is proved.

Now we return to the space $G\left({ }^{\mu} 2\right)$.

Let $\mathcal{U}_{\xi}$ be an arbitrary ultrafilter in $R O\left({ }^{\mu} 2\right)$ such that $\mathscr{Q}_{\xi} \supset \mathcal{\vartheta}_{\xi} \cup \mathscr{F}_{\xi}$, and let $p_{\xi}$ be the point in $G\left({ }^{\mu} 2\right)$ corresponding to $\mathcal{Q}_{\xi}$. Then the set $\left\{p_{\xi}: \xi \in \nu\right\}$ is a discrete subspace of $G\left({ }^{\mu} 2\right)$ by (a); furthermore, (ii) follows from (c) and from the definition of $\mathscr{F}_{\xi}$.

If $U \in \mathcal{U}_{p}$, then $U \in \mathscr{F}_{\xi}$ for all $\xi \in \nu$ such that there is an $A \in \mathbb{Q}(U)$ with $A \cap \nu \subset \xi$. Since $\nu$ is regular and uncountable, there is some $\alpha<\nu$ such that $U \in \mathscr{F}_{\xi}$ for all $\xi>\alpha$. But according to the definition of the topology in the Stone space, this means precisely (i).

If $q \neq p, q \in G\left({ }^{\mu} 2\right)$, then there are disjoint regular open sets $U, V$ in ${ }^{\mu}{ }_{2}$ corresponding to disjoint neighborhoods of $p$ and $q$. According to the definition of $\mathscr{F}_{\xi}, V$ cannot belong to $\mathscr{U}_{\xi}$ whenever $U \in \mathscr{F}_{\xi}$, which proves (iii).

3.5. THEOREM. Let $\mu$ be an infinite cardinal, let $X$ be a topological space containing a copy of $G\left({ }^{\mu} 2\right)$ as a subspace. Then for each regular $\nu, \omega_{1}<\nu<\mu$, there is a discrete set $D \subset X$ with $|D|=\nu$ and a point $q \in X$ such that $q$ is the unique complete accumulation point of $D$, and $q \notin \operatorname{cl}\left(D_{0}\right)$ for any $D_{0} \subset D$ with $\left|D_{0}\right|<\nu$.

Proof. Apply Lemma 3.4.

3.6. THEOREM. Let $\kappa, \lambda$ be infinite cardinals, $\lambda$ regular and $\lambda \leqslant 2^{\kappa}$. Then there is a uniform filter $\mathscr{F}$ and an ultrafilter $\mathscr{U} \supset \mathscr{F}$ on $\mathscr{P}(\kappa)$ with $\chi(\mathcal{U}, \mathscr{F})=\lambda$ if and only if $\lambda>\omega$.

Proof. By Stone duality, there is a one-to-one correspondence between closed sets in $U(\kappa)$ (points in $U(\kappa)$ ) and uniform filters (uniform ultrafilters, resp.) on $\mathscr{P}(\kappa)$. Hence it suffices to find a closed set $K \subset U(\kappa)$ and a point $p \in K$ with $\chi(p, K)=\lambda$. 
First we shall show that $\lambda>\omega$ is necessary. Let $p \in K \subset U(\kappa)$ be such that $\chi(p, K)=\omega$. Let $\left\{V_{n}: n \in \omega\right\}$ be the neighborhood base of $p$ in $K$. Let $p_{n} \in$ $\cap\left\{V_{i}: i<n\right\}, p_{n} \neq p$. The set $\left\{p_{n}: n \in \omega\right\}$ is $C^{*}$-embedded in $U(\kappa)$ (see, e.g., [CN, 16.15(b)]), yet it converges to $p$-a contradiction.

Let $\lambda$ be uncountable. According to Efimov's theorem, $G\left({ }^{2} 2\right)$ can be embedded into $U(\kappa)$. Since $\lambda \leqslant 2^{\kappa}$ and $\lambda$ is regular, 3.4 may be applied: let $p$ and $\left\{p_{\xi}: \xi \in \lambda\right\}$ be as stated in 3.4. Let $K=\operatorname{cl}\left(p_{\xi}: \xi \in \lambda\right)$. Then $\chi(p, K) \leqslant \lambda$ by 3.4(ii), (iii), since $\left\{K-\operatorname{cl}\left(p_{\xi}: \xi<\alpha\right): \alpha<\lambda\right\}$ is a neighborhood system of cardinality $\lambda$ of $p$ in $K$ whose intersection is $\{p\}$. On the other hand, 3.4(i) and the regularity of $\lambda$ imply that $\chi(p, K) \geqslant \lambda$.

Notice that an analogous theorem holds for any Boolean algebra $\mathscr{B}$, whose Stone space contains a copy of $G\left({ }^{\mu} 2\right)$ for some uncountable $\mu$. An interesting consequence of this fact can be found in $\left[\mathbf{v D}_{2}\right]$.

3.7. Corollary. (a) For each infinite cardinal $\kappa$, there is a discrete set $D \subset U(\kappa)$ of cardinality $\omega_{1}$ having a unique complete accumulation point.

(b) For each infinite cardinal $\kappa$, there exist a uniform filter $\mathcal{F}$ and a family $\mathcal{C [ \kappa ] ^ { \kappa }}$ such that $|\bigodot|=\omega_{1}$ and $\mathcal{F} \cup \bigodot$ generates a uniform ultrafilter on $\mathcal{P}(\kappa)$.

\section{REFERENCES}

[B] J. E. Baumgartner, Almost disjoint sets, the dense set problem and the partition calculus, Ann. Math. Logic 10 (1976), 401-439.

[BHM] J. Baumgartner, A. Hajnal and A. Máté, Weak saturation properties of ideals, Colloq. Math. Soc. János Bolyai 10, Infinite and Finite Sets, Keszthely, 1973, pp. 137-158.

[BF] B. Balcar and F. Franĕk, Independent families on complete Boolean algebras (to appear).

[BFM] B. Balcar, R. Frankiewicz and Ch. Mills, More on nowhere dense closed P-sets, Bull. Acad. Polon. Sci. Sér. Sci. Math. (to appear).

[BPS] B. Balcar, J. Pelant and P. Simon, The space of ultrafilters on $N$ covered by nowhere dense sets, Fund. Math. 110 (1980), 11-24.

[BV $\left.\mathbf{B}_{1}\right]$ B. Balcar and P. Vojtás, Refining systems on Boolean algebras, Lecture Notes in Math., vol. 619, Springer-Verlag, Berlin, 1977, pp. 45-58.

$\left[\mathrm{BV}_{2}\right] \ldots$ Almost disjoint refinement of families of subsets of N, Proc. Amer. Math. Soc. 79 (1980), 465-470.

[BK] M. Benda and J. Ketonen, Regularity of ultrafilters, Israel J. Math. 17 (1974), 231-240.

[C] W. W. Comfort, Compactifications. Recent results from several countries, Topology Proc. 2 (1977), 61-87.

[CH] W. W. Comfort and N. B. Hindman, Refining families for ultrafilters, Math. Z. 149 (1976), $189-199$.

[CN] W. W. Comfort and S. Negrepontis, The theory of ultrafilters, Springer-Verlag, Berlin, 1974.

[Ch] C. C. Chang, Descendingly incomplete ultrafilters, Trans. Amer. Math. Soc. 126 (1967), 108-118.

[vD $]$ E. K. van Douwen, Martin's axiom and pathological points in $\beta X-X$ (unpublished manuscript).

$\left[\mathbf{v D}_{2}\right] \ldots$, Cardinal functions on compact F-spaces and on weakly countably complete Boolean algebras (preprint).

[E] B. Efimov, Extremally disconnected bicompacta, Dokl. Akad. Nauk SSSR 172 (1967), 771-774.

[ET] P. Erdös and A. Tarski, On families of mutually exclusive sets, Ann. of Math. (2) 44 (1943), 315-329.

[H] S. H. Hechler, Generalizations of almost disjointness, c-sets, and the Baire number of $\beta N-N$, General Topology Appl. 8 (1978), 93-110.

[Hi] N. B. Hindman, On the existence of c-point in $\beta N-N$, Proc. Amer. Math. Soc. 21 (1969), 277-280.

[Hu] M. Hušek, Topological spaces without $\kappa$-accessible diagonal, Comment. Math. Univ. Carolinae 18 (4) (1977), 777-787. 
[J] T. J. Jech, Set theory, Academic Press, New York, 1978.

$\left[\mathbf{K}_{1}\right]$ H. J. Keisler, Good ideals in fields of sets, Ann. of Math. (2) 79 (1964), 338-359.

$\left[\mathbf{K}_{2}\right] \ldots$, Ultraproducts and saturated models, Indag. Math. 26 (1964), 178-186.

[Ku $]$ K. Kunen, Saturated ideals, J. Symbolic Logic 43 (1978), 65-76.

$\left[\mathrm{Ku}_{2}\right]$ _ Letter to the author, Sept. 14, 1978.

[KvMM] K. Kunen, J. van Mill and Ch. F. Mills, On nowhere dense closed P-sets, Proc. Amer. Math. Soc. 78 (1980), 119-123.

[KP] K. Kunen and K. Prikry, On descendingly incomplete ultrafilters, J. Symbolic Logic 36 (1971), $650-652$.

[Kr] K. Kuratowski, Sur l'extension de deux théorèmes topologiques à la théorie des ensembles, Fund. Math. 34 (1947), 34-38.

[L] R. Laver, Saturated ideals and nonregular ultrafilters (to appear).

$\left[\mathbf{P}_{1}\right]$ K. Prikry, Ultrafilters and almost disjoint sets, General Topology Appl. 4 (1974), 269-282.

$\left[\mathbf{P}_{2}\right]$ _ Ultrafilters and almost disjoint sets. II, Bull. Amer. Math. Soc. 81 (1975), 209-212.

$\left[\mathbf{P}_{\mathbf{3}}\right] \ldots$, On refinements of ultrafilters (preprint).

[S] S. Shelah, Some consistency results in topology (manuscript).

[Si] W. Sierpiński, Hypothèse du continu, 2nd ed., Chelsea, New York, 1956.

[T] A. D. Taylor, Regularity properties of ideals and ultrafilters, Ann. Math. Logic 16 (1979), 33-55.

[U] S. Ulam, Zur Masstheorie in der. allgemeinen Mengenlehre, Fund. Math. 16 (1930), 140-150.

[VW] R. Van Wesep, The non-stationary ideal on $\omega_{1}$ can be $\omega_{2}$-saturated (manuscript).

[VH] P. Vopénka and P. Hájek, The theory of semisets, North-Holland, Amsterdam, 1972.

[W] H. Woodin, An $\aleph_{1}$ dense $\aleph_{1}$ complete ideal on $\aleph_{1}$ (manuscript).

[Wo] R. G. Woods, Characterization of some $C^{*}$-embedded subspace of $\beta N$, Pacific J. Math. 65 (1976), 573-579.

ĆKD Praha O. P. závod Polovodiče, 14004 Prague, Czechoslovakia

Matematický ústav University Karlovy, Sokolovská 83, 18600 Prague, Czechoslovakia

Matematický ústav Slovenskej AKadémie Vied, Komenského 14; 04154 Košíce, CzechoSLOVAKIA 